\title{
Forecasting Portfolio Balance Using Return Mean, Standard Deviation and Spending
}

\author{
Jeffry Haber $^{1} \&$ Andrew Braunstein ${ }^{1}$ \\ ${ }^{1}$ Iona College, New Rochelle, NY, USA \\ Correspondence: Jeffry Haber, Iona College, 715 North Avenue, New Rochelle, NY 10801, USA
}

Received: February 18, 2016

Accepted: March 9, 2016

Online Published: March 21, 2016

doi:10.5430/ijfr.v7n2p98

URL: http://dx.doi.org/10.5430/ijfr.v7n2p98

\begin{abstract}
This paper develops an integrated formula using return mean, standard deviation and spending to forecast the ending balance of a portfolio. The forecasted ending balances were robust when tested over a variety of time periods, spending percentages, and varying how the spending was calculated.

This formula is useful for a variety of stakeholders - for government regulators to see how a change in required spending percentages would affect the long-term viability of institutions, for those institutions in understanding how the standard deviation (as a proxy for volatility) affects the portfolio balance, and for investment committees in understanding the trade-off between return and volatility and the resultant effect on the portfolio, among others.
\end{abstract}

Keywords: portfolio management, endowment, foundation, forecasting, portfolio balance, volatility, investment, return, standard deviation

\section{Introduction}

There are three principal metrics that will determine the ending balance of an endowment portfolio:

\section{Return}

2. Standard deviation of return

3. Rate of spending

Currently there appears to be a lack of research clearly demonstrating the specific interaction of those three variables, although all certainly are recognized as being critical to the management of an endowment portfolio. Conceptually there is a fourth variable that should be considered, namely capital additions (new money) that could flow into the endowment. For a college, university, museum, or pension, potential inflows are an important element that should be taken into account. For a private foundation or retiree, however, the omission of inflows is a reasonable assumption.

The motivation for this research was a problem devised as "food for thought" for the institutional investor community (Haber (2014a)). The problem was as follows:

Let's say you had an endowment with a balance of \$500,000,000. While on vacation, you find a lamp and rub it, and out pops a genie who grants you some wishes. With your first wish, you learn that over the next ten years you will experience five years of annual returns of $+5 \%$ and five years of annual returns of $-5 \%$. With your second wish, you can select the order in which the returns happen (five years of $+5 \%$ followed by five years of $-5 \%$, or five years of $-5 \%$ followed by five years of $+5 \%$, alternating years of positive and negative or negative and positive, and so on).

Without using a spreadsheet, what do you choose?

The answer is that the order of the returns does not matter. You will always wind up with the same ending portfolio balance. The problem was further expanded by introducing spending into the mix (Haber (2014b)):

For simplicity, let both the annual return and the annual spending be apportioned evenly throughout the year, and assume that the annual return and the annual spending occur at the same time of year (as opposed to spending happening at the beginning of the year and return at the end of the year, for instance).

Using a single spending factor for the 10 years, which of the following arrangements will produce the highest ending portfolio balance?: 
1. Positive returns first, negative returns follow

2. Negative returns first, positive returns follow

3. Alternating positive and negative, starting with positive

4. Alternating negative and positive, starting with negative

The answer, again, is that is does not matter. You will always arrive at the same ending endowment balance. This basic situation clearly is not often repeated in practice, given that returns can be highly volatile on a month-to-month basis. If spending is based on a past balance (such as prior month or prior year), then it would seem that there is the potential to be spending more (based on a higher previous endowment balance) than is warranted (based on a lower current endowment balance), and vice versa.

This paper incorporates spending using two mechanisms:

1. Based on $1 / 12$ of the previous month's endowment balance

2. Based on $1 / 12$ of the endowment balance at the previous year-end

There certainly are other common ways to incorporate a spending pattern (rolling 12-quarter periods, for example), so there is room for future research with a greater variety of spending rules.

The specific focus of this research is to develop a single, robust equation to predict a portfolio balance, utilizing a set of relevant variables. Such an equation would be extremely useful in shedding light on the interaction of the critical elements in the behavior of a portfolio, and thus provide insight for portfolio management. For example, consider an endowment whose spending is based on prior balances. One might well speculate that volatile returns could lead to spending that ultimately cannot be sustained, resulting in a negative spiral for the endowment. The type of equation presented in this study can help provide evidence as to whether such fears are indeed legitimate.

\section{Process}

Rather than using manufactured returns, we used the actual S\&P 500 monthly returns for the 10 year period from May 1, 2005 through April 30, 2015 (see Figure 1):

\begin{tabular}{cccc}
$\mathrm{N}$ & Month End & S\&P 500 & $\begin{array}{c}\text { S\&P 500 } \\
\text { Monthly Return }\end{array}$ \\
0 & $05 / 31 / 05$ & 1191.50 & \\
1 & $06 / 30 / 05$ & 1191.33 & -0.0143 \\
2 & $07 / 29 / 05$ & 1234.18 & 3.5968 \\
3 & $08 / 31 / 05$ & 1220.33 & -1.1222 \\
4 & $09 / 30 / 05$ & 1228.81 & 0.6949 \\
5 & $10 / 31 / 05$ & 1207.01 & -1.7741 \\
6 & $11 / 30 / 05$ & 1249.48 & 3.5186 \\
7 & $12 / 30 / 05$ & 1248.29 & -0.0952 \\
8 & $01 / 31 / 06$ & 1280.08 & 2.5467 \\
9 & $02 / 28 / 06$ & 1280.66 & 0.0453 \\
10 & $03 / 31 / 06$ & 1294.83 & 1.1065 \\
11 & $04 / 28 / 06$ & 1310.61 & 1.2187 \\
12 & $05 / 31 / 06$ & 1270.09 & -3.0917 \\
13 & $06 / 30 / 06$ & 1270.20 & 0.0087 \\
14 & $07 / 31 / 06$ & 1276.66 & 0.5086 \\
15 & $08 / 31 / 06$ & 1303.82 & 2.1274 \\
16 & $09 / 29 / 06$ & 1335.85 & 2.4566 \\
17 & $10 / 31 / 06$ & 1377.94 & 3.1508 \\
18 & $11 / 30 / 06$ & 1400.63 & 1.6467 \\
19 & $12 / 29 / 06$ & 1418.30 & 1.2616 \\
20 & $01 / 31 / 07$ & 1438.24 & 1.4059 \\
21 & $02 / 28 / 07$ & 1406.82 & -2.1846 \\
22 & $03 / 30 / 07$ & 1420.86 & 0.9980 \\
23 & $04 / 30 / 07$ & 1482.37 & 4.3291 \\
24 & $05 / 31 / 07$ & 1530.62 & 3.2549 \\
25 & $06 / 29 / 07$ & 1503.35 & -1.7816 \\
& & & \\
\hline & & &
\end{tabular}




\begin{tabular}{|c|c|c|c|}
\hline 26 & $07 / 31 / 07$ & 1455.27 & -3.1982 \\
\hline 27 & $08 / 31 / 07$ & 1473.99 & 1.2864 \\
\hline 28 & $09 / 28 / 07$ & 1526.75 & 3.5794 \\
\hline 29 & $10 / 31 / 07$ & 1549.38 & 1.4822 \\
\hline 30 & $11 / 30 / 07$ & 1481.14 & -4.4043 \\
\hline 31 & $12 / 31 / 07$ & 1468.36 & -0.8628 \\
\hline 32 & $01 / 31 / 08$ & 1378.55 & -6.1163 \\
\hline 33 & $02 / 28 / 08$ & 1367.68 & -0.7885 \\
\hline 34 & $03 / 31 / 08$ & 1322.70 & -3.2888 \\
\hline 35 & $04 / 30 / 08$ & 1385.59 & 4.7547 \\
\hline 36 & $05 / 30 / 08$ & 1400.38 & 1.0674 \\
\hline 37 & 06/30/08 & 1280.00 & -8.5962 \\
\hline 38 & $07 / 31 / 08$ & 1267.38 & -0.9859 \\
\hline 39 & 08/29/08 & 1282.83 & 1.2191 \\
\hline 40 & 09/30/08 & 1166.36 & -9.0791 \\
\hline 41 & $10 / 31 / 08$ & 968.75 & -16.9425 \\
\hline 42 & $11 / 28 / 08$ & 896.24 & -7.4849 \\
\hline 43 & $12 / 31 / 08$ & 903.25 & 0.7822 \\
\hline 44 & 01/30/09 & 825.88 & -8.5657 \\
\hline 45 & $02 / 27 / 09$ & 735.09 & -10.9931 \\
\hline 46 & $03 / 31 / 09$ & 797.87 & 8.5405 \\
\hline 47 & 04/30/09 & 872.81 & 9.3925 \\
\hline 48 & $05 / 29 / 09$ & 919.14 & 5.3081 \\
\hline 49 & 06/30/09 & 919.32 & 0.0196 \\
\hline 50 & 07/31/09 & 987.48 & 7.4142 \\
\hline 51 & 08/31/09 & 1020.62 & 3.3560 \\
\hline 52 & 09/30/09 & 1057.08 & 3.5723 \\
\hline 53 & $10 / 30 / 09$ & 1036.19 & -1.9762 \\
\hline 54 & $11 / 30 / 09$ & 1095.63 & 5.7364 \\
\hline 55 & $12 / 31 / 09$ & 1115.10 & 1.7771 \\
\hline 56 & $01 / 29 / 10$ & 1073.87 & -3.6974 \\
\hline 57 & $02 / 26 / 10$ & 1104.49 & 2.8514 \\
\hline 58 & $03 / 31 / 10$ & 1169.43 & 5.8796 \\
\hline 59 & $04 / 30 / 10$ & 1186.69 & 1.4759 \\
\hline 60 & $05 / 28 / 10$ & 1089.41 & -8.1976 \\
\hline 61 & $06 / 30 / 10$ & 1030.71 & -5.3882 \\
\hline 62 & $07 / 30 / 10$ & 1101.60 & 6.8778 \\
\hline 63 & $08 / 31 / 10$ & 1049.33 & -4.7449 \\
\hline 64 & $09 / 30 / 10$ & 1141.20 & 8.7551 \\
\hline 65 & $10 / 29 / 10$ & 1183.26 & 3.6856 \\
\hline 66 & $11 / 30 / 10$ & 1180.55 & -0.2290 \\
\hline 67 & $12 / 31 / 10$ & 1257.64 & 6.5300 \\
\hline 68 & $01 / 31 / 11$ & 1286.12 & 2.2646 \\
\hline 69 & $02 / 28 / 11$ & 1327.22 & 3.1957 \\
\hline 70 & $03 / 31 / 11$ & 1325.83 & -0.1047 \\
\hline 71 & $04 / 29 / 11$ & 1363.61 & 2.8495 \\
\hline 72 & $05 / 31 / 11$ & 1345.20 & -1.3501 \\
\hline 73 & $06 / 30 / 11$ & 1320.64 & -1.8258 \\
\hline 74 & 07/29/11 & 1292.28 & -2.1474 \\
\hline 75 & $08 / 31 / 11$ & 1218.89 & -5.6791 \\
\hline 76 & 09/30/11 & 1131.42 & -7.1762 \\
\hline 77 & $10 / 31 / 11$ & 1253.30 & 10.7723 \\
\hline 78 & $11 / 30 / 11$ & 1246.96 & -0.5059 \\
\hline 79 & $12 / 30 / 11$ & 1257.60 & 0.8533 \\
\hline 80 & $01 / 31 / 12$ & 1312.41 & 4.3583 \\
\hline
\end{tabular}




$\begin{array}{cccc}81 & 02 / 29 / 12 & 1365.68 & 4.0589 \\ 82 & 03 / 30 / 12 & 1408.47 & 3.1332 \\ 83 & 04 / 30 / 12 & 1397.91 & -0.7497 \\ 84 & 05 / 31 / 12 & 1310.33 & -6.2651 \\ 85 & 06 / 29 / 12 & 1362.16 & 3.9555 \\ 86 & 07 / 31 / 12 & 1379.32 & 1.2598 \\ 87 & 08 / 31 / 12 & 1406.58 & 1.9763 \\ 88 & 09 / 28 / 12 & 1440.67 & 2.4236 \\ 89 & 10 / 31 / 12 & 1412.16 & -1.9789 \\ 90 & 11 / 30 / 12 & 1416.18 & 0.2847 \\ 91 & 12 / 31 / 12 & 1426.19 & 0.7068 \\ 92 & 01 / 31 / 13 & 1498.11 & 5.0428 \\ 93 & 02 / 28 / 13 & 1514.68 & 1.1061 \\ 94 & 03 / 28 / 13 & 1569.19 & 3.5988 \\ 95 & 04 / 30 / 13 & 1597.57 & 1.8086 \\ 96 & 05 / 31 / 13 & 1630.74 & 2.0763 \\ 97 & 06 / 28 / 13 & 1606.28 & -1.4999 \\ 98 & 07 / 31 / 13 & 1685.73 & 4.9462 \\ 99 & 08 / 30 / 13 & 1632.97 & -3.1298 \\ 100 & 09 / 30 / 13 & 1681.55 & 2.9749 \\ 101 & 10 / 31 / 13 & 1756.54 & 4.4596 \\ 102 & 11 / 29 / 13 & 1805.81 & 2.8049 \\ 103 & 12 / 31 / 13 & 1848.36 & 2.3563 \\ 104 & 01 / 31 / 14 & 1782.59 & -3.5583 \\ 105 & 02 / 28 / 14 & 1859.45 & 4.3117 \\ 106 & 03 / 31 / 14 & 1872.34 & 0.6932 \\ 107 & 04 / 30 / 14 & 1883.95 & 0.6201 \\ 108 & 05 / 30 / 14 & 1923.57 & 2.1030 \\ 109 & 06 / 30 / 14 & 1960.23 & 1.9058 \\ 110 & 07 / 31 / 14 & 1930.67 & -1.5080 \\ 111 & 08 / 29 / 14 & 2003.37 & 3.7655 \\ 112 & 09 / 30 / 14 & 1972.29 & -1.5514 \\ 113 & 10 / 31 / 14 & 2018.05 & 2.3201 \\ 114 & 11 / 28 / 14 & 2067.56 & 2.4534 \\ 115 & 12 / 31 / 14 & 2058.90 & -0.4189 \\ 116 & 01 / 30 / 15 & 1994.99 & -3.1041 \\ 117 & 02 / 27 / 15 & 2104.50 & 5.4893 \\ 118 & 03 / 31 / 15 & 2067.89 & -1.7396 \\ 119 & 04 / 30 / 15 & 2085.51 & 0.8521\end{array}$

Figure 1. Monthly S\&P 500 returns

The arithmetic mean return during this period was $0.5639 \%$, and the compound mean return was $0.4715 \%$. The standard deviation of the actual monthly returns was $4.2677 \%$. We utilized a starting endowment balance of $\$ 500,000,000$ and four spending rates $(0 \%, 2 \%, 5 \%, 8 \%)$, applied according to two schemes (one based on the prior month's ending endowment balance and one based on the prior year's ending endowment balance) (the tables follow the list below).

Figure $2-0 \%$ spending

Figure $3-2 \%$ spending, based on prior month's endowment balance

Figure $4-5 \%$ spending, based on prior month's endowment balance

Figure $5-8 \%$ spending, based on prior month's endowment balance

Figure $6-2 \%$ spending, based on prior year's endowment balance 
Figure $7-5 \%$ spending, based on prior year's endowment balance

Figure $8-8 \%$ spending, based on prior year's endowment balance

\begin{tabular}{|c|c|}
\hline Month End & $\begin{array}{l}\text { Apply Monthly } \\
\text { S\&P } 500 \text { Returns }\end{array}$ \\
\hline $05 / 31 / 05$ & $500,000,000$ \\
\hline 06/30/05 & $499,928,661$ \\
\hline $07 / 29 / 05$ & $517,910,197$ \\
\hline 08/31/05 & $512,098,196$ \\
\hline $09 / 30 / 05$ & $515,656,735$ \\
\hline $10 / 31 / 05$ & $506,508,603$ \\
\hline $11 / 30 / 05$ & $524,330,676$ \\
\hline $12 / 30 / 05$ & $523,831,305$ \\
\hline $01 / 31 / 06$ & $537,171,632$ \\
\hline $02 / 28 / 06$ & $537,415,023$ \\
\hline 03/31/06 & $543,361,309$ \\
\hline $04 / 28 / 06$ & $549,983,214$ \\
\hline $05 / 31 / 06$ & $532,979,438$ \\
\hline 06/30/06 & $533,025,598$ \\
\hline $07 / 31 / 06$ & $535,736,467$ \\
\hline 08/31/06 & $547,133,865$ \\
\hline 09/29/06 & $560,574,906$ \\
\hline $10 / 31 / 06$ & $578,237,516$ \\
\hline $11 / 30 / 06$ & $587,759,127$ \\
\hline $12 / 29 / 06$ & $595,174,150$ \\
\hline $01 / 31 / 07$ & $603,541,754$ \\
\hline $02 / 28 / 07$ & $590,356,693$ \\
\hline 03/30/07 & $596,248,426$ \\
\hline $04 / 30 / 07$ & $622,060,428$ \\
\hline $05 / 31 / 07$ & $642,308,015$ \\
\hline 06/29/07 & $630,864,457$ \\
\hline $07 / 31 / 07$ & $610,688,208$ \\
\hline $08 / 31 / 07$ & $618,543,852$ \\
\hline 09/28/07 & $640,684,012$ \\
\hline $10 / 31 / 07$ & $650,180,445$ \\
\hline $11 / 30 / 07$ & $621,544,272$ \\
\hline $12 / 31 / 07$ & $616,181,284$ \\
\hline $01 / 31 / 08$ & $578,493,496$ \\
\hline $02 / 28 / 08$ & $573,932,018$ \\
\hline 03/31/08 & $555,056,651$ \\
\hline 04/30/08 & $581,447,755$ \\
\hline 05/30/08 & $587,654,217$ \\
\hline 06/30/08 & $537,138,061$ \\
\hline 07/31/08 & $531,842,216$ \\
\hline 08/29/08 & $538,325,640$ \\
\hline 09/30/08 & $489,450,273$ \\
\hline $10 / 31 / 08$ & $406,525,388$ \\
\hline $11 / 28 / 08$ & $376,097,356$ \\
\hline $12 / 31 / 08$ & $379,039,026$ \\
\hline 01/30/09 & $346,571,548$ \\
\hline 02/27/09 & $308,472,514$ \\
\hline 03/31/09 & $334,817,457$ \\
\hline 04/30/09 & $366,265,212$ \\
\hline
\end{tabular}




\begin{tabular}{|c|c|}
\hline 05/29/09 & $385,707,092$ \\
\hline 06/30/09 & $385,782,627$ \\
\hline 07/31/09 & $414,385,229$ \\
\hline 08/31/09 & $428,292,069$ \\
\hline 09/30/09 & $443,592,111$ \\
\hline $10 / 30 / 09$ & $434,825,850$ \\
\hline $11 / 30 / 09$ & $459,769,198$ \\
\hline $12 / 31 / 09$ & $467,939,572$ \\
\hline $01 / 29 / 10$ & $450,637,851$ \\
\hline $02 / 26 / 10$ & $463,487,201$ \\
\hline $03 / 31 / 10$ & $490,738,565$ \\
\hline $04 / 30 / 10$ & $497,981,536$ \\
\hline $05 / 28 / 10$ & $457,159,043$ \\
\hline $06 / 30 / 10$ & $432,526,227$ \\
\hline $07 / 30 / 10$ & $462,274,444$ \\
\hline $08 / 31 / 10$ & $440,339,908$ \\
\hline $09 / 30 / 10$ & $478,892,153$ \\
\hline $10 / 29 / 10$ & $496,542,174$ \\
\hline $11 / 30 / 10$ & $495,404,952$ \\
\hline $12 / 31 / 10$ & $527,754,931$ \\
\hline $01 / 31 / 11$ & $539,706,253$ \\
\hline $02 / 28 / 11$ & $556,953,420$ \\
\hline $03 / 31 / 11$ & $556,370,122$ \\
\hline $04 / 29 / 11$ & $572,224,087$ \\
\hline $05 / 31 / 11$ & $564,498,531$ \\
\hline $06 / 30 / 11$ & $554,192,195$ \\
\hline $07 / 29 / 11$ & $542,291,230$ \\
\hline $08 / 31 / 11$ & $511,493,915$ \\
\hline $09 / 30 / 11$ & $474,788,082$ \\
\hline $10 / 31 / 11$ & $525,933,697$ \\
\hline $11 / 30 / 11$ & $523,273,185$ \\
\hline $12 / 30 / 11$ & $527,738,145$ \\
\hline $01 / 31 / 12$ & $550,738,565$ \\
\hline $02 / 29 / 12$ & $573,092,740$ \\
\hline 03/30/12 & $591,049,098$ \\
\hline $04 / 30 / 12$ & $586,617,709$ \\
\hline $05 / 31 / 12$ & $549,865,715$ \\
\hline $06 / 29 / 12$ & $571,615,611$ \\
\hline $07 / 31 / 12$ & $578,816,618$ \\
\hline 08/31/12 & $590,255,980$ \\
\hline 09/28/12 & $604,561,477$ \\
\hline 10/31/12 & $592,597,566$ \\
\hline $11 / 30 / 12$ & $594,284,515$ \\
\hline $12 / 31 / 12$ & $598,485,103$ \\
\hline $01 / 31 / 13$ & $628,665,548$ \\
\hline $02 / 28 / 13$ & $635,618,968$ \\
\hline $03 / 28 / 13$ & $658,493,496$ \\
\hline $04 / 30 / 13$ & $670,402,854$ \\
\hline $05 / 31 / 13$ & $684,322,283$ \\
\hline $06 / 28 / 13$ & $674,057,910$ \\
\hline $07 / 31 / 13$ & $707,398,238$ \\
\hline $08 / 30 / 13$ & $685,258,078$ \\
\hline $09 / 30 / 13$ & $705,644,146$ \\
\hline $10 / 31 / 13$ & $737,112,883$ \\
\hline $11 / 29 / 13$ & $757,788,502$ \\
\hline
\end{tabular}




$\begin{array}{ll}12 / 31 / 13 & 775,644,146 \\ 01 / 31 / 14 & 748,044,482 \\ 02 / 28 / 14 & 780,297,944 \\ 03 / 31 / 14 & 785,707,092 \\ 04 / 30 / 14 & 790,579,102 \\ 05 / 30 / 14 & 807,205,204 \\ 06 / 30 / 14 & 822,589,173 \\ 07 / 31 / 14 & 810,184,641 \\ 08 / 29 / 14 & 840,692,405 \\ 09 / 30 / 14 & 827,650,021 \\ 10 / 31 / 14 & 846,852,707 \\ 11 / 28 / 14 & 867,629,039 \\ 12 / 31 / 14 & 863,994,964 \\ 01 / 30 / 15 & 837,175,829 \\ 02 / 27 / 15 & 883,130,508 \\ 03 / 31 / 15 & 867,767,520 \\ 04 / 30 / 15 & 875,161,561\end{array}$

Figure 2. 0\% Spending

Month End

05/31/05

$06 / 30 / 05$

07/29/05

$08 / 31 / 05$

$09 / 30 / 05$

$10 / 31 / 05$

$11 / 30 / 05$

$12 / 30 / 05$

$01 / 31 / 06$

$02 / 28 / 06$

$03 / 31 / 06$

$04 / 28 / 06$

$05 / 31 / 06$

$06 / 30 / 06$

$07 / 31 / 06$

$08 / 31 / 06$

$09 / 29 / 06$

$10 / 31 / 06$

$11 / 30 / 06$

$12 / 29 / 06$

$01 / 31 / 07$

$02 / 28 / 07$

$03 / 30 / 07$

$04 / 30 / 07$

$05 / 31 / 07$

$06 / 29 / 07$

$07 / 31 / 07$

08/31/07

$09 / 28 / 07$

$10 / 31 / 07$

$11 / 30 / 07$

$12 / 31 / 07$

Spend 2\%/12 of Prior Month's Balance

Spend

831,826

860,358

849,270

853,756

837,186

865,248

862,982

883,521

882,449

890,742

900,113

870,784

869,408

872,381

889,486

909,855

937,007

950,874

961,285

973,198

950,316

958,216

998,101

$1,028,924$

$1,008,878$

974,931

985,847

$1,019,491$

$1,032,903$

985,689
Return

$$
\begin{array}{r}
-71,339 \\
17,951,562 \\
-5,792,979 \\
3,540,914 \\
-9,087,754 \\
17,674,406 \\
-494,436 \\
13,186,459 \\
240,192 \\
5,858,373 \\
6,513,248 \\
-16,697,228 \\
45,250 \\
2,652,990 \\
11,135,559 \\
13,110,821 \\
17,200,648 \\
9,257,594 \\
7,197,596 \\
8,108,877 \\
-12,756,377 \\
5,690,464 \\
24,889,089 \\
19,492,442 \\
-10,998,982 \\
-19,359,504 \\
7,524,666 \\
21,172,442 \\
9,066,744 \\
-27,295,558 \\
-5,103,005
\end{array}
$$

104
Balance

$500,000,000$ $499,095,328$

$516,215,065$

$509,561,728$

$512,253,372$

$502,311,862$

$519,149,081$

$517,789,397$

$530,112,874$

$529,469,545$

$534,445,468$

$540,067,974$

$522,470,633$

$521,645,099$

$523,428,680$

$533,691,858$

$545,913,192$

$562,203,985$

$570,524,572$

$576,771,294$

$583,918,885$

$570,189,310$

$574,929,458$

$598,860,331$

$617,354,672$

$605,326,766$

$584,958,384$

$591,508,119$

$611,694,715$

$619,741,967$

$591,413,506$

$585,324,812$ 


\begin{tabular}{|c|c|c|c|}
\hline 01/31/08 & 975,541 & $-35,800,499$ & $548,548,771$ \\
\hline $02 / 28 / 08$ & 914,248 & $-4,325,360$ & $543,309,163$ \\
\hline 03/31/08 & 905,515 & $-17,868,249$ & $524,535,399$ \\
\hline 04/30/08 & 874,226 & $24,939,919$ & $548,601,093$ \\
\hline 05/30/08 & 914,335 & $5,855,852$ & $553,542,610$ \\
\hline 06/30/08 & 922,571 & $-47,583,841$ & $505,036,198$ \\
\hline 07/31/08 & 841,727 & $-4,979,341$ & $499,215,130$ \\
\hline 08/29/08 & 832,025 & $6,085,684$ & $504,468,788$ \\
\hline 09/30/08 & 840,781 & $-45,801,454$ & $457,826,552$ \\
\hline $10 / 31 / 08$ & 763,044 & $-77,567,050$ & $379,496,458$ \\
\hline $11 / 28 / 08$ & 632,494 & $-28,404,943$ & $350,459,021$ \\
\hline $12 / 31 / 08$ & 584,098 & $2,741,138$ & $352,616,061$ \\
\hline 01/30/09 & 587,693 & $-30,204,157$ & $321,824,211$ \\
\hline $02 / 27 / 09$ & 536,374 & $-35,378,530$ & $285,909,307$ \\
\hline 03/31/09 & 476,516 & $24,417,944$ & $309,850,735$ \\
\hline 04/30/09 & 516,418 & $29,102,754$ & $338,437,071$ \\
\hline 05/29/09 & 564,062 & $17,964,723$ & $355,837,732$ \\
\hline 06/30/09 & 593,063 & 69,686 & $355,314,355$ \\
\hline 07/31/09 & 592,191 & $26,343,631$ & $381,065,795$ \\
\hline 08/31/09 & 635,110 & $12,788,634$ & $393,219,319$ \\
\hline 09/30/09 & 655,366 & $14,047,125$ & $406,611,078$ \\
\hline 10/30/09 & 677,685 & $-8,035,442$ & $397,897,951$ \\
\hline 11/30/09 & 663,163 & $22,825,017$ & $420,059,804$ \\
\hline $12 / 31 / 09$ & 700,100 & $7,464,714$ & $426,824,419$ \\
\hline 01/29/10 & 711,374 & $-15,781,518$ & $410,331,526$ \\
\hline $02 / 26 / 10$ & 683,886 & $11,700,067$ & $421,347,708$ \\
\hline $03 / 31 / 10$ & 702,246 & $24,773,715$ & $445,419,177$ \\
\hline $04 / 30 / 10$ & 742,365 & $6,574,087$ & $451,250,899$ \\
\hline $05 / 28 / 10$ & 752,085 & $-36,991,706$ & $413,507,108$ \\
\hline 06/30/10 & 689,179 & $-22,280,746$ & $390,537,184$ \\
\hline 07/30/10 & 650,895 & $26,860,301$ & $416,746,589$ \\
\hline $08 / 31 / 10$ & 694,578 & $-19,774,278$ & $396,277,734$ \\
\hline $09 / 30 / 10$ & 660,463 & $34,694,553$ & $430,311,824$ \\
\hline $10 / 29 / 10$ & 717,186 & $15,859,547$ & $445,454,185$ \\
\hline $11 / 30 / 10$ & 742,424 & $-1,020,216$ & $443,691,546$ \\
\hline $12 / 31 / 10$ & 739,486 & $28,973,090$ & $471,925,150$ \\
\hline $01 / 31 / 11$ & 786,542 & $10,687,024$ & $481,825,631$ \\
\hline $02 / 28 / 11$ & 803,043 & $15,397,501$ & $496,420,089$ \\
\hline $03 / 31 / 11$ & 827,367 & $-519,902$ & $495,072,821$ \\
\hline 04/29/11 & 825,121 & $14,107,277$ & $508,354,976$ \\
\hline $05 / 31 / 11$ & 847,258 & $-6,863,264$ & $500,644,454$ \\
\hline $06 / 30 / 11$ & 834,407 & $-9,140,520$ & $490,669,527$ \\
\hline 07/29/11 & 817,783 & $-10,536,852$ & $479,314,892$ \\
\hline 08/31/11 & 798,858 & $-27,220,819$ & $451,295,215$ \\
\hline 09/30/11 & 752,159 & $-32,385,853$ & $418,157,203$ \\
\hline 10/31/11 & 696,929 & $45,045,164$ & $462,505,439$ \\
\hline 11/30/11 & 770,842 & $-2,339,651$ & $459,394,946$ \\
\hline $12 / 30 / 11$ & 765,658 & $3,919,903$ & $462,549,191$ \\
\hline $01 / 31 / 12$ & 770,915 & $20,159,288$ & $481,937,564$ \\
\hline $02 / 29 / 12$ & 803,229 & $19,561,581$ & $500,695,915$ \\
\hline 03/30/12 & 834,493 & $15,687,993$ & $515,549,415$ \\
\hline $04 / 30 / 12$ & 859,249 & $-3,865,330$ & $510,824,835$ \\
\hline $05 / 31 / 12$ & 851,375 & $-32,003,519$ & $477,969,942$ \\
\hline $06 / 29 / 12$ & 796,617 & $18,906,063$ & $496,079,389$ \\
\hline 07/31/12 & 826,799 & $6,249,429$ & $501,502,019$ \\
\hline
\end{tabular}




$\begin{array}{lrrr}08 / 31 / 12 & & & \\ 09 / 28 / 12 & 835,837 & 9,911,366 & 510,577,548 \\ 10 / 31 / 12 & 850,963 & 12,374,404 & 522,100,989 \\ 11 / 30 / 12 & 851,498 & -10,332,067 & 510,898,753 \\ 12 / 31 / 12 & 1,454,377 & 511,501,632 \\ 01 / 31 / 13 & 852,503 & 3,615,452 & 514,264,582 \\ 02 / 28 / 13 & 857,108 & 25,933,367 & 539,340,842 \\ 03 / 28 / 13 & 898,901 & 5,965,435 & 544,407,375 \\ 04 / 30 / 13 & 907,346 & 19,592,023 & 563,092,053 \\ 05 / 31 / 13 & 938,487 & 10,183,950 & 572,337,516 \\ 06 / 28 / 13 & 953,896 & 11,883,320 & 583,266,940 \\ 07 / 31 / 13 & 972,112 & -8,748,611 & 573,546,218 \\ 08 / 30 / 13 & 955,910 & 28,368,807 & 600,959,114 \\ 09 / 30 / 13 & 1,001,599 & -18,808,826 & 581,148,689 \\ 10 / 31 / 13 & 968,581 & 17,288,868 & 597,468,977 \\ 11 / 29 / 13 & 995,782 & 26,644,583 & 623,117,778 \\ 12 / 31 / 13 & 1,038,530 & 17,478,118 & 639,557,366 \\ 01 / 31 / 14 & 1,065,929 & 15,069,784 & 653,561,221 \\ 02 / 28 / 14 & 1,089,269 & -23,255,600 & 629,216,352 \\ 03 / 31 / 14 & 1,048,694 & 27,129,945 & 655,297,603 \\ 04 / 30 / 14 & 1,092,163 & 4,542,626 & 658,748,066 \\ 05 / 30 / 14 & 1,097,913 & 4,084,763 & 661,734,916 \\ 06 / 30 / 14 & 1,102,892 & 13,916,472 & 674,548,496 \\ 07 / 31 / 14 & 1,124,247 & 12,855,757 & 686,280,005 \\ 08 / 29 / 14 & 1,143,800 & -10,349,009 & 674,787,197 \\ 09 / 30 / 14 & 1,124,645 & 25,409,329 & 699,071,881 \\ 10 / 31 / 14 & 1,165,120 & -10,845,303 & 687,061,458 \\ 11 / 28 / 14 & 1,145,102 & 15,940,826 & 701,857,182 \\ 12 / 31 / 14 & 1,169,762 & 17,219,072 & 717,906,492 \\ 01 / 30 / 15 & 1,196,511 & -3,006,960 & 713,703,022 \\ 02 / 27 / 15 & 1,189,505 & -22,153,946 & 690,359,570 \\ 03 / 31 / 15 & 1,150,599 & 37,895,567 & 727,104,538 \\ 04 / 30 / 15 & 1,211,841 & -12,648,751 & 713,243,945 \\ & 1,188,740 & 6,077,382 & 718,132,588\end{array}$

Figure 3. 2\% Spending, based on prior month's endowment balance

\begin{tabular}{crrr} 
& \multicolumn{3}{c}{ Spend $\% / 12$ of Prior Month's Balance } \\
Month End & \multicolumn{1}{l}{ Spend } & \multicolumn{1}{l}{ Balance } \\
& & & \\
$05 / 31 / 05$ & & & $500,000,000$ \\
$06 / 30 / 05$ & $2,083,333$ & $-71,339$ & $497,845,328$ \\
$07 / 29 / 05$ & $2,074,356$ & $17,906,602$ & $513,677,575$ \\
$08 / 31 / 05$ & $2,140,323$ & $-5,764,503$ & $505,772,748$ \\
$09 / 30 / 05$ & $2,107,386$ & $3,514,585$ & $507,179,946$ \\
$10 / 31 / 05$ & $2,113,250$ & $-8,997,748$ & $496,068,948$ \\
$11 / 30 / 05$ & $2,066,954$ & $17,454,742$ & $511,456,737$ \\
$12 / 30 / 05$ & $2,131,070$ & $-487,109$ & $508,838,557$ \\
$01 / 31 / 06$ & $2,120,161$ & $12,958,509$ & $519,676,906$ \\
$02 / 28 / 06$ & $2,165,320$ & 235,464 & $517,747,050$ \\
$03 / 31 / 06$ & $2,157,279$ & $5,728,668$ & $521,318,438$ \\
$04 / 28 / 06$ & $2,172,160$ & $6,353,270$ & $525,499,548$ \\
$05 / 31 / 06$ & $2,189,581$ & $-16,246,818$ & $507,063,149$ \\
$06 / 30 / 06$ & $2,112,763$ & 43,916 & $504,994,302$ \\
$07 / 31 / 06$ & $2,104,143$ & $2,568,307$ & $505,458,465$
\end{tabular}




\begin{tabular}{|c|c|c|c|}
\hline 08/31/06 & $2,106,077$ & $10,753,256$ & $514,105,645$ \\
\hline $09 / 29 / 06$ & $2,142,107$ & $12,629,660$ & $524,593,198$ \\
\hline $10 / 31 / 06$ & $2,185,805$ & $16,528,897$ & $538,936,291$ \\
\hline $11 / 30 / 06$ & $2,245,568$ & $8,874,453$ & $545,565,176$ \\
\hline $12 / 29 / 06$ & $2,273,188$ & $6,882,715$ & $550,174,703$ \\
\hline $01 / 31 / 07$ & $2,292,395$ & $7,734,953$ & $555,617,261$ \\
\hline $02 / 28 / 07$ & $2,315,072$ & $-12,138,095$ & $541,164,094$ \\
\hline 03/30/07 & $2,254,850$ & $5,400,793$ & $544,310,036$ \\
\hline $04 / 30 / 07$ & $2,267,958$ & $23,563,553$ & $565,605,631$ \\
\hline 05/31/07 & $2,356,690$ & $18,410,027$ & $581,658,968$ \\
\hline 06/29/07 & $2,423,579$ & $-10,363,016$ & $568,872,373$ \\
\hline 07/31/07 & $2,370,302$ & $-18,193,623$ & $548,308,448$ \\
\hline 08/31/07 & $2,284,619$ & $7,053,216$ & $553,077,046$ \\
\hline 09/28/07 & $2,304,488$ & $19,796,840$ & $570,569,398$ \\
\hline $10 / 31 / 07$ & $2,377,372$ & $8,457,171$ & $576,649,197$ \\
\hline $11 / 30 / 07$ & $2,402,705$ & $-25,397,605$ & $548,848,887$ \\
\hline $12 / 31 / 07$ & $2,286,870$ & $-4,735,737$ & $541,826,280$ \\
\hline 01/31/08 & $2,257,609$ & $-33,139,978$ & $506,428,692$ \\
\hline $02 / 28 / 08$ & $2,110,120$ & $-3,993,239$ & $500,325,334$ \\
\hline 03/31/08 & $2,084,689$ & $-16,454,605$ & $481,786,040$ \\
\hline $04 / 30 / 08$ & $2,007,442$ & $22,907,329$ & $502,685,927$ \\
\hline 05/30/08 & $2,094,525$ & $5,365,747$ & $505,957,149$ \\
\hline 06/30/08 & $2,108,155$ & $-43,493,282$ & $460,355,713$ \\
\hline 07/31/08 & $1,918,149$ & $-4,538,820$ & $453,898,744$ \\
\hline 08/29/08 & $1,891,245$ & $5,533,254$ & $457,540,754$ \\
\hline 09/30/08 & $1,906,420$ & $-41,540,790$ & $414,093,544$ \\
\hline $10 / 31 / 08$ & $1,725,390$ & $-70,157,606$ & $342,210,548$ \\
\hline $11 / 28 / 08$ & $1,425,877$ & $-25,614,128$ & $315,170,543$ \\
\hline $12 / 31 / 08$ & $1,313,211$ & $2,465,127$ & $316,322,459$ \\
\hline 01/30/09 & $1,318,010$ & $-27,095,343$ & $287,909,106$ \\
\hline $02 / 27 / 09$ & $1,199,621$ & $-31,650,201$ & $255,059,284$ \\
\hline 03/31/09 & $1,062,747$ & $21,783,213$ & $275,779,750$ \\
\hline 04/30/09 & $1,149,082$ & $25,902,634$ & $300,533,301$ \\
\hline 05/29/09 & $1,252,222$ & $15,952,736$ & $315,233,815$ \\
\hline 06/30/09 & $1,313,474$ & 61,734 & $313,982,075$ \\
\hline 07/31/09 & $1,308,259$ & $23,279,183$ & $335,952,999$ \\
\hline 08/31/09 & $1,399,804$ & $11,274,641$ & $345,827,836$ \\
\hline 09/30/09 & $1,440,949$ & $12,354,141$ & $356,741,027$ \\
\hline $10 / 30 / 09$ & $1,486,421$ & $-7,049,911$ & $348,204,695$ \\
\hline $11 / 30 / 09$ & $1,450,853$ & $19,974,413$ & $366,728,255$ \\
\hline $12 / 31 / 09$ & $1,528,034$ & $6,516,980$ & $371,717,201$ \\
\hline $01 / 29 / 10$ & $1,548,822$ & $-13,743,969$ & $356,424,410$ \\
\hline $02 / 26 / 10$ & $1,485,102$ & $10,162,976$ & $365,102,285$ \\
\hline $03 / 31 / 10$ & $1,521,260$ & $21,466,688$ & $385,047,713$ \\
\hline $04 / 30 / 10$ & $1,604,365$ & $5,683,045$ & $389,126,393$ \\
\hline $05 / 28 / 10$ & $1,621,360$ & $-31,898,993$ & $355,606,041$ \\
\hline 06/30/10 & $1,481,692$ & $-19,160,899$ & $334,963,450$ \\
\hline $07 / 30 / 10$ & $1,395,681$ & $23,038,060$ & $356,605,829$ \\
\hline $08 / 31 / 10$ & $1,485,858$ & $-16,920,649$ & $338,199,323$ \\
\hline 09/30/10 & $1,409,164$ & $29,609,724$ & $366,399,883$ \\
\hline $10 / 29 / 10$ & $1,526,666$ & $13,504,013$ & $378,377,229$ \\
\hline $11 / 30 / 10$ & $1,576,572$ & $-866,591$ & $375,934,067$ \\
\hline $12 / 31 / 10$ & $1,566,392$ & $24,548,522$ & $398,916,196$ \\
\hline $01 / 31 / 11$ & $1,662,151$ & $9,033,693$ & $406,287,738$ \\
\hline $02 / 28 / 11$ & $1,692,866$ & $12,983,568$ & $417,578,440$ \\
\hline
\end{tabular}




\begin{tabular}{|c|c|c|c|}
\hline $03 / 31 / 11$ & $1,739,910$ & $-437,331$ & $415,401,199$ \\
\hline $04 / 29 / 11$ & $1,730,838$ & $11,837,006$ & $425,507,367$ \\
\hline $05 / 31 / 11$ & $1,772,947$ & $-5,744,744$ & $417,989,675$ \\
\hline 06/30/11 & $1,741,624$ & $-7,631,450$ & $408,616,602$ \\
\hline 07/29/11 & $1,702,569$ & $-8,774,811$ & $398,139,221$ \\
\hline $08 / 31 / 11$ & $1,658,913$ & $-22,610,763$ & $373,869,544$ \\
\hline 09/30/11 & $1,557,790$ & $-26,829,631$ & $345,482,123$ \\
\hline $10 / 31 / 11$ & $1,439,509$ & $37,216,384$ & $381,258,999$ \\
\hline 11/30/11 & $1,588,579$ & $-1,928,654$ & $377,741,766$ \\
\hline $12 / 30 / 11$ & $1,573,924$ & $3,223,177$ & $379,391,018$ \\
\hline $01 / 31 / 12$ & $1,580,796$ & $16,535,005$ & $394,345,227$ \\
\hline $02 / 29 / 12$ & $1,643,105$ & $16,006,256$ & $408,708,378$ \\
\hline 03/30/12 & $1,702,952$ & $12,805,805$ & $419,811,231$ \\
\hline $04 / 30 / 12$ & $1,749,213$ & $-3,147,534$ & $414,914,484$ \\
\hline $05 / 31 / 12$ & $1,728,810$ & $-25,994,671$ & $387,191,002$ \\
\hline 06/29/12 & $1,613,296$ & $15,315,310$ & $400,893,016$ \\
\hline $07 / 31 / 12$ & $1,670,388$ & $5,050,306$ & $404,272,934$ \\
\hline $08 / 31 / 12$ & $1,684,471$ & $7,989,792$ & $410,578,256$ \\
\hline 09/28/12 & $1,710,743$ & $9,950,812$ & $418,818,325$ \\
\hline 10/31/12 & $1,745,076$ & $-8,288,165$ & $408,785,084$ \\
\hline $11 / 30 / 12$ & $1,703,271$ & $1,163,690$ & $408,245,502$ \\
\hline $12 / 31 / 12$ & $1,701,023$ & $2,885,606$ & $409,430,085$ \\
\hline $01 / 31 / 13$ & $1,705,959$ & $20,646,766$ & $428,370,893$ \\
\hline $02 / 28 / 13$ & $1,784,879$ & $4,738,040$ & $431,324,054$ \\
\hline $03 / 28 / 13$ & $1,797,184$ & $15,522,404$ & $445,049,274$ \\
\hline $04 / 30 / 13$ & $1,854,372$ & $8,049,056$ & $451,243,959$ \\
\hline $05 / 31 / 13$ & $1,880,183$ & $9,369,081$ & $458,732,856$ \\
\hline $06 / 28 / 13$ & $1,911,387$ & $-6,880,683$ & $449,940,786$ \\
\hline $07 / 31 / 13$ & $1,874,753$ & $22,255,021$ & $470,321,054$ \\
\hline 08/30/13 & $1,959,671$ & $-14,720,115$ & $453,641,268$ \\
\hline 09/30/13 & $1,890,172$ & $13,495,590$ & $465,246,686$ \\
\hline $10 / 31 / 13$ & $1,938,528$ & $20,748,029$ & $484,056,187$ \\
\hline $11 / 29 / 13$ & $2,016,901$ & $13,577,515$ & $495,616,801$ \\
\hline $12 / 31 / 13$ & $2,065,070$ & $11,678,136$ & $505,229,868$ \\
\hline $01 / 31 / 14$ & $2,105,124$ & $-17,977,541$ & $485,147,202$ \\
\hline $02 / 28 / 14$ & $2,021,447$ & $20,918,110$ & $504,043,865$ \\
\hline $03 / 31 / 14$ & $2,100,183$ & $3,494,111$ & $505,437,794$ \\
\hline $04 / 30 / 14$ & 2,105,991 & $3,134,117$ & $506,465,920$ \\
\hline $05 / 30 / 14$ & $2,110,275$ & $10,651,121$ & $515,006,767$ \\
\hline $06 / 30 / 14$ & $2,145,862$ & $9,815,160$ & $522,676,065$ \\
\hline $07 / 31 / 14$ & $2,177,817$ & $-7,881,884$ & $512,616,365$ \\
\hline $08 / 29 / 14$ & $2,135,902$ & $19,302,734$ & $529,783,198$ \\
\hline 09/30/14 & $2,207,430$ & $-8,218,982$ & $519,356,786$ \\
\hline $10 / 31 / 14$ & $2,163,987$ & $12,049,834$ & $529,242,633$ \\
\hline $11 / 28 / 14$ & $2,205,178$ & $12,984,219$ & $540,021,674$ \\
\hline $12 / 31 / 14$ & $2,250,090$ & $-2,261,887$ & $535,509,696$ \\
\hline $01 / 30 / 15$ & $2,231,290$ & $-16,622,675$ & $516,655,731$ \\
\hline $02 / 27 / 15$ & $2,152,732$ & $28,360,528$ & $542,863,527$ \\
\hline $03 / 31 / 15$ & $2,261,931$ & $-9,443,684$ & $531,157,911$ \\
\hline $04 / 30 / 15$ & $2,213,158$ & $4,525,871$ & $533,470,624$ \\
\hline
\end{tabular}

Figure 4. 5\% Spending, based on prior month's endowment balance 
Month End

$05 / 31 / 05$

$06 / 30 / 05$

$07 / 29 / 05$

$08 / 31 / 05$

$09 / 30 / 05$

$10 / 31 / 05$

$11 / 30 / 05$

$12 / 30 / 05$

$01 / 31 / 06$

$02 / 28 / 06$

$03 / 31 / 06$

$04 / 28 / 06$

$05 / 31 / 06$

$06 / 30 / 06$

$07 / 31 / 06$

$08 / 31 / 06$

$09 / 29 / 06$

$10 / 31 / 06$

$11 / 30 / 06$

$12 / 29 / 06$

$01 / 31 / 07$

$02 / 28 / 07$

$03 / 30 / 07$

04/30/07

05/31/07

06/29/07

07/31/07

08/31/07

$09 / 28 / 07$

$10 / 31 / 07$

$11 / 30 / 07$

$12 / 31 / 07$

$01 / 31 / 08$

$02 / 28 / 08$

$03 / 31 / 08$

04/30/08

05/30/08

$06 / 30 / 08$

07/31/08

08/29/08

09/30/08

$10 / 31 / 08$

$11 / 28 / 08$

$12 / 31 / 08$

$01 / 30 / 09$

$02 / 27 / 09$

03/31/09

04/30/09

05/29/09

06/30/09

07/31/09

08/31/09

Spend $8 \% / 12$ of Prior Month's Balance

Spend

$3,333,333$

$3,310,636$

$3,407,642$

$3,346,684$

$3,347,629$

$3,265,922$

$3,359,064$

$3,333,471$

$3,396,141$

$3,375,039$

$3,389,882$

$3,408,595$

$3,280,488$

$3,258,902$

$3,253,750$

$3,301,280$

$3,360,371$

$3,443,847$

$3,477,597$

$3,498,286$

$3,524,146$

$3,423,663$

$3,435,007$

$3,560,810$

$3,652,973$

$3,563,538$

$3,425,812$

$3,447,041$

$3,547,445$

$3,576,376$

$3,395,018$

$3,343,091$

$3,116,328$

$3,070,980$

$2,949,509$

$3,070,085$

$3,082,389$

$2,796,870$

$2,750,649$

$2,765,843$

$2,496,289$

$2,056,714$

$1,889,060$

$1,891,242$

$1,716,635$

$1,516,479$

$1,635,883$

$1,778,627$

$1,861,182$

$1,849,139$

$1,973,909$
Return

$-71,339$

$17,861,642$

$-5,736,097$

$3,488,386$

$-8,908,412$

$17,237,267$

$-479,874$

$12,733,946$

230,817

$5,601,522$

$6,196,837$

$-15,807,479$

42,617

$2,486,125$

$10,383,170$

$12,165,021$

$15,881,801$

$8,506,274$

$6,580,875$

$7,377,404$

$-11,548,352$

$5,125,200$

$22,305,567$

$17,385,244$

$-9,762,375$

$-17,095,309$

$6,610,237$

$18,507,511$

$7,887,212$

$-23,627,379$

$-4,394,081$

$-30,671,256$

$-3,685,882$

$-15,149,672$

$21,035,908$

$4,915,584$

$-39,745,419$

$-4,136,308$

$5,029,769$

$-37,667,233$

$-63,439,888$

$-23,091,462$

$2,216,311$

$-24,299,811$

$-28,306,761$

$19,427,116$

$23,047,563$

$14,161,812$

54,673

$20,564,757$

$9,936,711$
Balance

$500,000,000$

$496,595,328$

$511,146,334$

$502,002,595$

$502,144,297$

$489,888,256$

$503,859,602$

$500,020,664$

$509,421,138$

$506,255,814$

$508,482,297$

$511,289,253$

$492,073,178$

$488,835,308$

$488,062,531$

$495,191,951$

$504,055,693$

$516,577,123$

$521,639,549$

$524,742,827$

$528,621,945$

$513,549,447$

$515,250,985$

$534,121,545$

$547,945,979$

$534,530,631$

$513,871,784$

$517,056,209$

$532,116,679$

$536,456,446$

$509,252,691$

$501,463,592$

$467,449,245$

$460,647,035$

$442,426,382$

$460,512,781$

$462,358,279$

$419,530,472$

$412,597,294$

$414,876,414$

$374,443,339$

$308,507,161$

$283,358,985$

$283,686,236$

$257,495,183$

$227,471,788$

$245,382,425$

$266,794,105$

$279,177,290$

$277,370,781$

$296,086,399$

$304,049,201$ 


\begin{tabular}{|c|c|c|c|}
\hline 09/30/09 & $2,026,995$ & $10,861,666$ & $312,883,872$ \\
\hline $10 / 30 / 09$ & $2,085,892$ & $-6,183,207$ & $304,614,773$ \\
\hline 11/30/09 & $2,030,765$ & $17,473,921$ & $320,057,929$ \\
\hline $12 / 31 / 09$ & $2,133,720$ & $5,687,621$ & $323,611,830$ \\
\hline $01 / 29 / 10$ & $2,157,412$ & $-11,965,309$ & $309,489,109$ \\
\hline $02 / 26 / 10$ & $2,063,261$ & $8,824,678$ & $316,250,526$ \\
\hline $03 / 31 / 10$ & $2,108,337$ & $18,594,382$ & $332,736,571$ \\
\hline $04 / 30 / 10$ & $2,218,244$ & $4,910,968$ & $335,429,295$ \\
\hline $05 / 28 / 10$ & $2,236,195$ & $-27,497,124$ & $305,695,976$ \\
\hline 06/30/10 & $2,037,973$ & $-16,471,626$ & $287,186,377$ \\
\hline $07 / 30 / 10$ & $1,914,576$ & $19,752,057$ & $305,023,858$ \\
\hline $08 / 31 / 10$ & $2,033,492$ & $-14,473,127$ & $288,517,238$ \\
\hline 09/30/10 & $1,923,448$ & $25,260,003$ & $311,853,793$ \\
\hline $10 / 29 / 10$ & $2,079,025$ & $11,493,665$ & $321,268,433$ \\
\hline $11 / 30 / 10$ & $2,141,790$ & $-735,796$ & $318,390,848$ \\
\hline $12 / 31 / 10$ & $2,122,606$ & $20,790,945$ & $337,059,187$ \\
\hline $01 / 31 / 11$ & $2,247,061$ & $7,632,904$ & $342,445,030$ \\
\hline $02 / 28 / 11$ & $2,282,967$ & $10,943,373$ & $351,105,436$ \\
\hline $03 / 31 / 11$ & $2,340,703$ & $-367,713$ & $348,397,020$ \\
\hline 04/29/11 & $2,322,647$ & $9,927,698$ & $356,002,071$ \\
\hline $05 / 31 / 11$ & $2,373,347$ & $-4,806,358$ & $348,822,365$ \\
\hline 06/30/11 & $2,325,482$ & $-6,368,627$ & $340,128,256$ \\
\hline $07 / 29 / 11$ & $2,267,522$ & $-7,304,063$ & $330,556,671$ \\
\hline $08 / 31 / 11$ & $2,203,711$ & $-18,772,676$ & $309,580,284$ \\
\hline 09/30/11 & $2,063,869$ & $-22,216,104$ & $285,300,311$ \\
\hline $10 / 31 / 11$ & $1,902,002$ & $30,733,416$ & $314,131,725$ \\
\hline $11 / 30 / 11$ & $2,094,212$ & $-1,589,081$ & $310,448,433$ \\
\hline $12 / 30 / 11$ & $2,069,656$ & $2,648,979$ & $311,027,756$ \\
\hline $01 / 31 / 12$ & $2,073,518$ & $13,555,527$ & $322,509,765$ \\
\hline $02 / 29 / 12$ & $2,150,065$ & $13,090,494$ & $333,450,194$ \\
\hline $03 / 30 / 12$ & $2,223,001$ & $10,447,787$ & $341,674,980$ \\
\hline $04 / 30 / 12$ & $2,277,833$ & $-2,561,707$ & $336,835,439$ \\
\hline $05 / 31 / 12$ & $2,245,570$ & $-21,102,966$ & $313,486,903$ \\
\hline $06 / 29 / 12$ & $2,089,913$ & $12,399,950$ & $323,796,940$ \\
\hline $07 / 31 / 12$ & $2,158,646$ & $4,079,077$ & $325,717,371$ \\
\hline $08 / 31 / 12$ & $2,171,449$ & $6,437,270$ & $329,983,192$ \\
\hline $09 / 28 / 12$ & $2,199,888$ & $7,997,502$ & $335,780,807$ \\
\hline $10 / 31 / 12$ & $2,238,539$ & $-6,644,902$ & $326,897,366$ \\
\hline $11 / 30 / 12$ & $2,179,316$ & 930,580 & $325,648,630$ \\
\hline $12 / 31 / 12$ & $2,170,991$ & $2,301,786$ & $325,779,425$ \\
\hline $01 / 31 / 13$ & $2,171,863$ & $16,428,426$ & $340,035,987$ \\
\hline $02 / 28 / 13$ & $2,266,907$ & $3,761,003$ & $341,530,084$ \\
\hline $03 / 28 / 13$ & $2,276,867$ & $12,290,916$ & $351,544,133$ \\
\hline $04 / 30 / 13$ & $2,343,628$ & $6,357,944$ & $355,558,450$ \\
\hline $05 / 31 / 13$ & $2,370,390$ & $7,382,383$ & $360,570,443$ \\
\hline $06 / 28 / 13$ & $2,403,803$ & $-5,408,313$ & $352,758,327$ \\
\hline $07 / 31 / 13$ & $2,351,722$ & $17,448,172$ & $367,854,776$ \\
\hline $08 / 30 / 13$ & $2,452,365$ & $-11,513,124$ & $353,889,287$ \\
\hline $09 / 30 / 13$ & $2,359,262$ & $10,528,020$ & $362,058,046$ \\
\hline $10 / 31 / 13$ & $2,413,720$ & $16,146,254$ & $375,790,579$ \\
\hline $11 / 29 / 13$ & $2,505,271$ & $10,540,723$ & $383,826,032$ \\
\hline $12 / 31 / 13$ & $2,558,840$ & $9,044,029$ & $390,311,220$ \\
\hline $01 / 31 / 14$ & $2,602,075$ & $-13,888,403$ & $373,820,742$ \\
\hline $02 / 28 / 14$ & $2,492,138$ & $16,118,043$ & $387,446,647$ \\
\hline $03 / 31 / 14$ & $2,582,978$ & $2,685,841$ & $387,549,510$ \\
\hline
\end{tabular}




$\begin{array}{lrrr}04 / 30 / 14 & 2,583,663 & 2,403,116 & 387,368,963 \\ 05 / 30 / 14 & 2,582,460 & 8,146,479 & 392,932,982 \\ 06 / 30 / 14 & 2,619,553 & 7,488,640 & 397,802,068 \\ 07 / 31 / 14 & 2,652,014 & -5,998,801 & 389,151,254 \\ 08 / 29 / 14 & 2,594,342 & 14,653,616 & 401,210,528 \\ 09 / 30 / 14 & 2,674,737 & -6,224,324 & 392,311,467 \\ 10 / 31 / 14 & 2,615,410 & 9,102,197 & 398,798,255 \\ 11 / 28 / 14 & 2,658,655 & 9,783,951 & 405,923,551 \\ 12 / 31 / 14 & 2,706,157 & -1,700,216 & 401,517,178 \\ 01 / 30 / 15 & 2,676,781 & -12,463,433 & 386,376,963 \\ 02 / 27 / 15 & 2,575,846 & 21,209,200 & 405,010,317 \\ 03 / 31 / 15 & 2,700,069 & -7,045,582 & 395,264,666 \\ 04 / 30 / 15 & 2,635,098 & 3,367,956 & 395,997,524\end{array}$

Figure 5. 8\% Spending, based on prior month's endowment balance

\begin{tabular}{|c|c|c|c|}
\hline \multirow[b]{2}{*}{ Month End } & \multicolumn{3}{|c|}{ Spend $2 \%$ of Prior Year End Balance } \\
\hline & Spend & Return & Balance \\
\hline 05/31/05 & & & \\
\hline $06 / 30 / 05$ & & & \\
\hline 07/29/05 & & & \\
\hline $08 / 31 / 05$ & & & \\
\hline 09/30/05 & & & \\
\hline $10 / 31 / 05$ & & & \\
\hline $11 / 30 / 05$ & & & \\
\hline $12 / 30 / 05$ & & & \\
\hline $01 / 31 / 06$ & & & \\
\hline $02 / 28 / 06$ & & & \\
\hline $03 / 31 / 06$ & & & \\
\hline $04 / 28 / 06$ & & & \\
\hline $05 / 31 / 06$ & & & \\
\hline $06 / 30 / 06$ & & & $533,025,598$ \\
\hline 07/31/06 & 888,376 & $2,710,869$ & $534,848,091$ \\
\hline $08 / 31 / 06$ & 888,376 & $11,378,499$ & $545,338,213$ \\
\hline 09/29/06 & 888,376 & $13,396,928$ & $557,846,766$ \\
\hline $10 / 31 / 06$ & 888,376 & $17,576,652$ & $574,535,041$ \\
\hline $11 / 30 / 06$ & 888,376 & $9,460,644$ & $583,107,310$ \\
\hline $12 / 29 / 06$ & 888,376 & $7,356,337$ & $589,575,271$ \\
\hline $01 / 31 / 07$ & 888,376 & $8,288,889$ & $596,975,783$ \\
\hline $02 / 28 / 07$ & 888,376 & $-13,041,620$ & $583,045,788$ \\
\hline 03/30/07 & 888,376 & $5,818,771$ & $587,976,182$ \\
\hline 04/30/07 & 888,376 & $25,453,891$ & $612,541,697$ \\
\hline $05 / 31 / 07$ & 888,376 & $19,937,760$ & $631,591,081$ \\
\hline $06 / 29 / 07$ & 888,376 & $-11,252,622$ & $619,450,082$ \\
\hline 07/31/07 & $1,032,417$ & $-19,811,195$ & $598,606,470$ \\
\hline 08/31/07 & $1,032,417$ & $7,700,230$ & $605,274,283$ \\
\hline 09/28/07 & $1,032,417$ & $21,665,188$ & $625,907,055$ \\
\hline $10 / 31 / 07$ & $1,032,417$ & $9,277,404$ & $634,152,042$ \\
\hline $11 / 30 / 07$ & $1,032,417$ & $-27,930,227$ & $605,189,398$ \\
\hline $12 / 31 / 07$ & $1,032,417$ & $-5,221,870$ & $598,935,111$ \\
\hline $01 / 31 / 08$ & $1,032,417$ & $-36,632,953$ & $561,269,742$ \\
\hline $02 / 28 / 08$ & $1,032,417$ & $-4,425,666$ & $555,811,659$ \\
\hline 03/31/08 & $1,032,417$ & $-18,279,428$ & $536,499,814$ \\
\hline
\end{tabular}




\begin{tabular}{|c|c|c|c|}
\hline 04/30/08 & $1,032,417$ & $25,508,788$ & $560,976,185$ \\
\hline 05/30/08 & $1,032,417$ & $5,987,946$ & $565,931,714$ \\
\hline 06/30/08 & $1,032,417$ & $-48,648,838$ & $516,250,459$ \\
\hline 07/31/08 & 860,417 & $-5,089,907$ & $510,300,135$ \\
\hline 08/29/08 & 860,417 & $6,220,815$ & $515,660,533$ \\
\hline 09/30/08 & 860,417 & $-46,817,569$ & $467,982,546$ \\
\hline $10 / 31 / 08$ & 860,417 & $-79,287,725$ & $387,834,404$ \\
\hline $11 / 28 / 08$ & 860,417 & $-29,029,030$ & $357,944,956$ \\
\hline $12 / 31 / 08$ & 860,417 & $2,799,690$ & $359,884,229$ \\
\hline 01/30/09 & 860,417 & $-30,826,729$ & $328,197,083$ \\
\hline 02/27/09 & 860,417 & $-36,079,107$ & $291,257,558$ \\
\hline 03/31/09 & 860,417 & $24,874,709$ & $315,271,849$ \\
\hline 04/30/09 & 860,417 & $29,611,932$ & $344,023,364$ \\
\hline 05/29/09 & 860,417 & $18,261,251$ & $361,424,197$ \\
\hline 06/30/09 & 860,417 & 70,780 & $360,634,560$ \\
\hline 07/31/09 & 601,058 & $26,738,080$ & $386,771,582$ \\
\hline 08/31/09 & 601,058 & $12,980,121$ & $399,150,646$ \\
\hline 09/30/09 & 601,058 & $14,259,012$ & $412,808,600$ \\
\hline 10/30/09 & 601,058 & $-8,157,918$ & $404,049,624$ \\
\hline $11 / 30 / 09$ & 601,058 & $23,177,901$ & $426,626,468$ \\
\hline $12 / 31 / 09$ & 601,058 & $7,581,407$ & $433,606,818$ \\
\hline 01/29/10 & 601,058 & $-16,032,292$ & $416,973,468$ \\
\hline $02 / 26 / 10$ & 601,058 & $11,889,454$ & $428,261,864$ \\
\hline $03 / 31 / 10$ & 601,058 & $25,180,242$ & $452,841,049$ \\
\hline $04 / 30 / 10$ & 601,058 & $6,683,629$ & $458,923,620$ \\
\hline $05 / 28 / 10$ & 601,058 & $-37,620,684$ & $420,701,878$ \\
\hline $06 / 30 / 10$ & 601,058 & $-22,668,417$ & $397,432,404$ \\
\hline $07 / 30 / 10$ & 662,387 & $27,334,539$ & $424,104,556$ \\
\hline 08/31/10 & 662,387 & $-20,123,407$ & $403,318,761$ \\
\hline 09/30/10 & 662,387 & $35,311,003$ & $437,967,377$ \\
\hline $10 / 29 / 10$ & 662,387 & $16,141,700$ & $453,446,689$ \\
\hline $11 / 30 / 10$ & 662,387 & $-1,038,521$ & $451,745,781$ \\
\hline $12 / 31 / 10$ & 662,387 & $29,499,032$ & $480,582,426$ \\
\hline $01 / 31 / 11$ & 662,387 & $10,883,073$ & $490,803,111$ \\
\hline $02 / 28 / 11$ & 662,387 & $15,684,390$ & $505,825,114$ \\
\hline $03 / 31 / 11$ & 662,387 & $-529,752$ & $504,632,975$ \\
\hline 04/29/11 & 662,387 & $14,379,697$ & $518,350,284$ \\
\hline $05 / 31 / 11$ & 662,387 & $-6,998,210$ & $510,689,687$ \\
\hline $06 / 30 / 11$ & 662,387 & $-9,323,921$ & $500,703,379$ \\
\hline 07/29/11 & 834,506 & $-10,752,323$ & $489,116,550$ \\
\hline $08 / 31 / 11$ & 834,506 & $-27,777,466$ & $460,504,579$ \\
\hline 09/30/11 & 834,506 & $-33,046,736$ & $426,623,338$ \\
\hline $10 / 31 / 11$ & 834,506 & $45,957,162$ & $471,745,994$ \\
\hline 11/30/11 & 834,506 & $-2,386,396$ & $468,525,093$ \\
\hline $12 / 30 / 11$ & 834,506 & $3,997,808$ & $471,688,395$ \\
\hline $01 / 31 / 12$ & 834,506 & $20,557,603$ & $491,411,492$ \\
\hline $02 / 29 / 12$ & 834,506 & $19,946,122$ & $510,523,109$ \\
\hline 03/30/12 & 834,506 & $15,995,902$ & $525,684,506$ \\
\hline 04/30/12 & 834,506 & $-3,941,318$ & $520,908,682$ \\
\hline $05 / 31 / 12$ & 834,506 & $-32,635,279$ & $487,438,898$ \\
\hline $06 / 29 / 12$ & 834,506 & $19,280,607$ & $505,884,999$ \\
\hline $07 / 31 / 12$ & 843,142 & $6,372,957$ & $511,414,814$ \\
\hline 08/31/12 & 843,142 & $10,107,276$ & $520,678,948$ \\
\hline 09/28/12 & 843,142 & $12,619,222$ & $532,455,029$ \\
\hline $10 / 31 / 12$ & 843,142 & $-10,536,967$ & $521,074,920$ \\
\hline
\end{tabular}




$\begin{array}{lrrr}11 / 30 / 12 & 843,142 & 1,483,345 & 521,715,123 \\ 12 / 31 / 12 & 843,142 & 3,687,644 & 524,559,626 \\ 01 / 31 / 13 & 843,142 & 26,452,526 & 550,169,011 \\ 02 / 28 / 13 & 843,142 & 6,085,201 & 555,411,070 \\ 03 / 28 / 13 & 843,142 & 19,988,022 & 574,555,951 \\ 04 / 30 / 13 & 843,142 & 10,391,283 & 584,104,092 \\ 05 / 31 / 13 & 843,142 & 12,127,627 & 595,388,578 \\ 06 / 28 / 13 & 843,142 & -8,930,427 & 585,615,009 \\ 07 / 31 / 13 & 976,025 & 28,965,755 & 613,604,738 \\ 08 / 30 / 13 & 976,025 & -19,204,609 & 593,424,104 \\ 09 / 30 / 13 & 976,025 & 17,654,055 & 610,102,135 \\ 10 / 31 / 13 & 976,025 & 27,207,968 & 636,334,078 \\ 11 / 29 / 13 & 976,025 & 17,848,828 & 653,206,881 \\ 12 / 31 / 13 & 976,025 & 15,391,405 & 667,622,261 \\ 01 / 31 / 14 & 976,025 & -23,755,933 & 642,890,303 \\ 02 / 28 / 14 & 976,025 & 27,719,525 & 669,633,803 \\ 03 / 31 / 14 & 976,025 & 4,642,007 & 673,299,785 \\ 04 / 30 / 14 & 976,025 & 4,174,995 & 676,498,755 \\ 05 / 30 / 14 & 976,025 & 14,226,960 & 689,749,690 \\ 06 / 30 / 14 & 976,025 & 13,145,466 & 701,919,130 \\ 07 / 31 / 14 & 1,169,865 & -10,584,844 & 690,164,421 \\ 08 / 29 / 14 & 1,169,865 & 25,988,363 & 714,982,919 \\ 09 / 30 / 14 & 1,169,865 & -11,092,144 & 702,720,909 \\ 10 / 31 / 14 & 1,169,865 & 16,304,148 & 717,855,193 \\ 11 / 28 / 14 & 1,169,865 & 17,611,561 & 734,296,888 \\ 12 / 31 / 14 & 1,169,865 & -3,075,611 & 730,051,412 \\ 01 / 30 / 15 & 1,169,865 & -22,661,414 & 706,220,132 \\ 02 / 27 / 15 & 1,169,865 & 38,766,193 & 743,816,460 \\ 03 / 31 / 15 & 1,169,865 & -12,939,473 & 729,707,122 \\ 04 / 30 / 15 & 1,169,865 & 6,217,661 & 734,754,918\end{array}$

Figure 6. 2\% Spending, based on prior year's endowment balance

\begin{tabular}{lccc} 
& \multicolumn{3}{c}{ Spend 5\% of Prior Year End Balance } \\
Month End & Spend & Return & Balance \\
& & & \\
$05 / 31 / 05$ & & & \\
$06 / 30 / 05$ & & & \\
$07 / 29 / 05$ & & & \\
$08 / 31 / 05$ & & & \\
$09 / 30 / 05$ & & & \\
$10 / 31 / 05$ & & & \\
$11 / 30 / 05$ & & & \\
$12 / 30 / 05$ & & & \\
$01 / 31 / 06$ & & & \\
$02 / 28 / 06$ & & & \\
$03 / 31 / 06$ & & & \\
$04 / 28 / 06$ & & & \\
$05 / 31 / 06$ & & & \\
$06 / 30 / 06$ & & & \\
$07 / 31 / 06$ & & & \\
$08 / 31 / 06$ & $2,220,940$ & & \\
$09 / 29 / 06$ & $2,220,940$ & $11,350,149,515,598$ \\
$10 / 31 / 06$ & $2,220,940$ & $13,330,760$ & $553,754,556$ \\
& $2,220,940$ & $17,447,714$ & $568,981,330$
\end{tabular}




\begin{tabular}{|c|c|c|c|}
\hline $11 / 30 / 06$ & $2,220,940$ & $9,369,193$ & $576,129,583$ \\
\hline $12 / 29 / 06$ & $2,220,940$ & $7,268,308$ & $581,176,951$ \\
\hline $01 / 31 / 07$ & $2,220,940$ & $8,170,816$ & $587,126,827$ \\
\hline $02 / 28 / 07$ & $2,220,940$ & $-12,826,458$ & $572,079,429$ \\
\hline 03/30/07 & $2,220,940$ & $5,709,327$ & $575,567,816$ \\
\hline $04 / 30 / 07$ & $2,220,940$ & $24,916,724$ & $598,263,600$ \\
\hline $05 / 31 / 07$ & $2,220,940$ & $19,473,019$ & $615,515,679$ \\
\hline 06/29/07 & $2,220,940$ & $-10,966,218$ & $602,328,521$ \\
\hline $07 / 31 / 07$ & $2,509,702$ & $-19,263,615$ & $580,555,204$ \\
\hline 08/31/07 & $2,509,702$ & $7,468,025$ & $585,513,527$ \\
\hline 09/28/07 & $2,509,702$ & $20,957,872$ & $603,961,697$ \\
\hline $10 / 31 / 07$ & $2,509,702$ & $8,952,123$ & $610,404,117$ \\
\hline $11 / 30 / 07$ & $2,509,702$ & $-26,884,287$ & $581,010,128$ \\
\hline $12 / 31 / 07$ & $2,509,702$ & $-5,013,239$ & $573,487,186$ \\
\hline $01 / 31 / 08$ & $2,509,702$ & $-35,076,469$ & $535,901,015$ \\
\hline $02 / 28 / 08$ & $2,509,702$ & $-4,225,631$ & $529,165,681$ \\
\hline 03/31/08 & $2,509,702$ & $-17,403,100$ & $509,252,879$ \\
\hline $04 / 30 / 08$ & $2,509,702$ & $24,213,286$ & $530,956,463$ \\
\hline 05/30/08 & $2,509,702$ & $5,667,511$ & $534,114,271$ \\
\hline 06/30/08 & $2,509,702$ & $-45,913,735$ & $485,690,834$ \\
\hline 07/31/08 & $2,023,712$ & $-4,788,608$ & $478,878,514$ \\
\hline 08/29/08 & $2,023,712$ & $5,837,770$ & $482,692,573$ \\
\hline 09/30/08 & $2,023,712$ & $-43,824,360$ & $436,844,501$ \\
\hline $10 / 31 / 08$ & $2,023,712$ & $-74,012,176$ & $360,808,613$ \\
\hline $11 / 28 / 08$ & $2,023,712$ & $-27,006,175$ & $331,778,725$ \\
\hline $12 / 31 / 08$ & $2,023,712$ & $2,595,029$ & $332,350,043$ \\
\hline 01/30/09 & $2,023,712$ & $-28,468,223$ & $301,858,108$ \\
\hline $02 / 27 / 09$ & $2,023,712$ & $-33,183,631$ & $266,650,764$ \\
\hline 03/31/09 & $2,023,712$ & $22,773,177$ & $287,400,230$ \\
\hline 04/30/09 & $2,023,712$ & $26,994,088$ & $312,370,606$ \\
\hline 05/29/09 & $2,023,712$ & $16,581,077$ & $326,927,972$ \\
\hline 06/30/09 & $2,023,712$ & 64,024 & $324,968,284$ \\
\hline 07/31/09 & $1,354,035$ & $24,093,720$ & $347,707,969$ \\
\hline 08/31/09 & $1,354,035$ & $11,669,140$ & $358,023,074$ \\
\hline 09/30/09 & $1,354,035$ & $12,789,796$ & $369,458,836$ \\
\hline $10 / 30 / 09$ & $1,354,035$ & $-7,301,240$ & $360,803,561$ \\
\hline 11/30/09 & $1,354,035$ & $20,697,134$ & $380,146,661$ \\
\hline $12 / 31 / 09$ & $1,354,035$ & $6,755,433$ & $385,548,059$ \\
\hline $01 / 29 / 10$ & $1,354,035$ & $-14,255,355$ & $369,938,670$ \\
\hline $02 / 26 / 10$ & $1,354,035$ & $10,548,318$ & $379,132,953$ \\
\hline $03 / 31 / 10$ & $1,354,035$ & $22,291,640$ & $400,070,559$ \\
\hline $04 / 30 / 10$ & $1,354,035$ & $5,904,772$ & $404,621,297$ \\
\hline $05 / 28 / 10$ & $1,354,035$ & $-33,169,202$ & $370,098,061$ \\
\hline $06 / 30 / 10$ & $1,354,035$ & $-19,941,763$ & $348,802,263$ \\
\hline 07/30/10 & $1,453,343$ & $23,989,864$ & $371,338,784$ \\
\hline 08/31/10 & $1,453,343$ & $-17,619,715$ & $352,265,726$ \\
\hline $09 / 30 / 10$ & $1,453,343$ & $30,841,253$ & $381,653,637$ \\
\hline $10 / 29 / 10$ & $1,453,343$ & $14,066,204$ & $394,266,498$ \\
\hline $11 / 30 / 10$ & $1,453,343$ & $-902,982$ & $391,910,173$ \\
\hline $12 / 31 / 10$ & $1,453,343$ & $25,591,763$ & $416,048,593$ \\
\hline $01 / 31 / 11$ & $1,453,343$ & $9,421,666$ & $424,016,916$ \\
\hline $02 / 28 / 11$ & $1,453,343$ & $13,550,132$ & $436,113,705$ \\
\hline 03/31/11 & $1,453,343$ & $-456,743$ & $434,203,620$ \\
\hline $04 / 29 / 11$ & $1,453,343$ & $12,372,787$ & $445,123,064$ \\
\hline $05 / 31 / 11$ & $1,453,343$ & $-6,009,574$ & $437,660,147$ \\
\hline
\end{tabular}




$\begin{array}{lrrr}06 / 30 / 11 & 1,453,343 & -7,990,584 & 428,216,221 \\ 07 / 29 / 11 & 1,784,234 & -9,195,702 & 417,236,284 \\ 08 / 31 / 11 & 1,784,234 & -23,695,307 & 391,756,743 \\ 09 / 30 / 11 & 1,784,234 & -28,113,253 & 361,859,257 \\ 10 / 31 / 11 & 1,784,234 & 38,980,579 & 399,055,601 \\ 11 / 30 / 11 & 1,784,234 & -2,018,681 & 395,252,686 \\ 12 / 30 / 11 & 1,784,234 & 3,372,593 & 396,841,045 \\ 01 / 31 / 12 & 1,784,234 & 17,295,529 & 412,352,340 \\ 02 / 29 / 12 & 1,784,234 & 16,737,155 & 427,305,260 \\ 03 / 30 / 12 & 1,784,234 & 13,388,489 & 438,909,515 \\ 04 / 30 / 12 & 1,784,234 & -3,290,723 & 433,834,558 \\ 05 / 31 / 12 & 1,784,234 & -27,180,026 & 404,870,298 \\ 06 / 29 / 12 & 1,784,234 & 16,014,613 & 419,100,676 \\ 07 / 31 / 12 & 1,746,253 & 5,279,679 & 422,634,102 \\ 08 / 31 / 12 & 1,746,253 & 8,352,671 & 429,240,520 \\ 09 / 28 / 12 & 1,746,253 & 10,403,112 & 437,897,379 \\ 10 / 31 / 12 & 1,746,253 & -8,665,728 & 427,485,399 \\ 11 / 30 / 12 & 1,746,253 & 1,216,924 & 426,956,070 \\ 12 / 31 / 12 & 1,746,253 & 3,017,858 & 428,227,675 \\ 01 / 31 / 13 & 1,746,253 & 21,594,692 & 448,076,115 \\ 02 / 28 / 13 & 1,746,253 & 4,955,992 & 451,285,854 \\ 03 / 28 / 13 & 1,746,253 & 16,240,785 & 465,780,386 \\ 04 / 30 / 13 & 1,746,253 & 8,423,994 & 472,458,127 \\ 05 / 31 / 13 & 1,746,253 & 9,809,546 & 480,521,420 \\ 06 / 28 / 13 & 1,746,253 & -7,207,497 & 471,567,670 \\ 07 / 31 / 13 & 1,964,865 & 23,324,733 & 492,927,537 \\ 08 / 30 / 13 & 1,964,865 & -15,427,653 & 475,535,019 \\ 09 / 30 / 13 & 1,964,865 & 14,146,917 & 487,717,071 \\ 10 / 31 / 13 & 1,964,865 & 21,750,113 & 507,502,319 \\ 11 / 29 / 13 & 1,964,865 & 14,235,166 & 519,772,620 \\ 12 / 31 / 13 & 1,964,865 & 12,247,316 & 530,055,071 \\ 01 / 31 / 14 & 1,964,865 & -18,860,894 & 509,229,311 \\ 02 / 28 / 14 & 1,964,865 & 21,956,459 & 529,220,906 \\ 03 / 31 / 14 & 1,964,865 & 3,668,643 & 530,924,683 \\ 04 / 30 / 14 & 1,964,865 & 3,292,156 & 532,251,974 \\ 05 / 30 / 14 & 1,964,865 & 11,193,409 & 541,480,518 \\ 06 / 30 / 14 & 1,964,865 & 10,319,705 & 549,835,358 \\ 07 / 31 / 14 & 2,290,981 & -8,291,442 & 539,252,935 \\ 08 / 29 / 14 & 2,290,981 & 20,305,743 & 557,267,697 \\ 09 / 30 / 14 & 2,290,981 & -8,645,373 & 546,331,344 \\ 10 / 31 / 14 & 2,290,981 & 12,675,683 & 556,716,046 \\ 11 / 28 / 14 & 2,290,981 & 13,658,240 & 568,083,305 \\ 12 / 31 / 14 & 2,290,981 & -2,379,424 & 563,412,901 \\ 01 / 30 / 15 & 2,290,981 & -17,488,814 & 543,633,107 \\ 02 / 27 / 15 & 2,290,981 & 29,841,383 & 571,183,509 \\ 03 / 31 / 15 & 2,290,981 & -9,936,340 & 558,956,188 \\ 04 / 30 / 15 & 2,290,981 & 4,762,733 & 561,427,941\end{array}$

Figure $7.5 \%$ Spending, based on prior year's endowment balance

Month End $\quad$ Spend $\frac{\text { Spend 8\% of Prior Year End Balance }}{\text { Return }}$ Balance

$05 / 31 / 05$ 


\begin{tabular}{|c|c|c|c|}
\hline \multicolumn{4}{|l|}{$06 / 30 / 05$} \\
\hline $07 / 29 / 05$ & & & \\
\hline 08/31/05 & & & \\
\hline 09/30/05 & & & \\
\hline $10 / 31 / 05$ & & & \\
\hline $11 / 30 / 05$ & & & \\
\hline $12 / 30 / 05$ & & & \\
\hline $01 / 31 / 06$ & & & \\
\hline $02 / 28 / 06$ & & & \\
\hline 03/31/06 & & & \\
\hline $04 / 28 / 06$ & & & \\
\hline 05/31/06 & & & \\
\hline 06/30/06 & & & $533,025,598$ \\
\hline 07/31/06 & $3,553,504$ & $2,710,869$ & $532,182,963$ \\
\hline 08/31/06 & $3,553,504$ & $11,321,800$ & $539,951,259$ \\
\hline 09/29/06 & $3,553,504$ & $13,264,591$ & $549,662,346$ \\
\hline $10 / 31 / 06$ & $3,553,504$ & $17,318,777$ & $563,427,619$ \\
\hline $11 / 30 / 06$ & $3,553,504$ & $9,277,743$ & $569,151,857$ \\
\hline $12 / 29 / 06$ & $3,553,504$ & $7,180,278$ & $572,778,632$ \\
\hline $01 / 31 / 07$ & $3,553,504$ & $8,052,743$ & $577,277,871$ \\
\hline $02 / 28 / 07$ & $3,553,504$ & $-12,611,296$ & $561,113,071$ \\
\hline 03/30/07 & $3,553,504$ & $5,599,883$ & $563,159,450$ \\
\hline 04/30/07 & $3,553,504$ & $24,379,557$ & $583,985,503$ \\
\hline 05/31/07 & $3,553,504$ & $19,008,278$ & $599,440,277$ \\
\hline 06/29/07 & $3,553,504$ & $-10,679,814$ & $585,206,959$ \\
\hline 07/31/07 & $3,901,380$ & $-18,716,035$ & $562,589,545$ \\
\hline 08/31/07 & $3,901,380$ & $7,236,923$ & $565,925,088$ \\
\hline 09/28/07 & $3,901,380$ & $20,256,723$ & $582,280,431$ \\
\hline $10 / 31 / 07$ & $3,901,380$ & $8,630,756$ & $587,009,807$ \\
\hline $11 / 30 / 07$ & $3,901,380$ & $-25,853,922$ & $557,254,506$ \\
\hline $12 / 31 / 07$ & $3,901,380$ & $-4,808,264$ & $548,544,862$ \\
\hline $01 / 31 / 08$ & $3,901,380$ & $-33,550,910$ & $511,092,572$ \\
\hline $02 / 28 / 08$ & $3,901,380$ & $-4,030,014$ & $503,161,178$ \\
\hline 03/31/08 & $3,901,380$ & $-16,547,869$ & $482,711,929$ \\
\hline $04 / 30 / 08$ & $3,901,380$ & $22,951,352$ & $501,761,901$ \\
\hline 05/30/08 & $3,901,380$ & $5,355,883$ & $503,216,405$ \\
\hline 06/30/08 & $3,901,380$ & $-43,257,681$ & $456,057,345$ \\
\hline 07/31/08 & $3,040,382$ & $-4,496,440$ & $448,520,522$ \\
\hline 08/29/08 & $3,040,382$ & $5,467,691$ & $450,947,830$ \\
\hline 09/30/08 & $3,040,382$ & $-40,942,209$ & $406,965,239$ \\
\hline $10 / 31 / 08$ & $3,040,382$ & $-68,949,896$ & $334,974,961$ \\
\hline $11 / 28 / 08$ & $3,040,382$ & $-25,072,552$ & $306,862,027$ \\
\hline $12 / 31 / 08$ & $3,040,382$ & $2,400,141$ & $306,221,786$ \\
\hline 01/30/09 & $3,040,382$ & $-26,230,146$ & $276,951,258$ \\
\hline $02 / 27 / 09$ & $3,040,382$ & $-30,445,591$ & $243,465,284$ \\
\hline 03/31/09 & $3,040,382$ & $20,793,033$ & $261,217,935$ \\
\hline 04/30/09 & $3,040,382$ & $24,534,914$ & $282,712,467$ \\
\hline 05/29/09 & $3,040,382$ & $15,006,781$ & $294,678,866$ \\
\hline 06/30/09 & $3,040,382$ & 57,709 & $291,696,192$ \\
\hline 07/31/09 & $1,944,641$ & $21,626,868$ & $311,378,419$ \\
\hline 08/31/09 & $1,944,641$ & $10,449,914$ & $319,883,691$ \\
\hline 09/30/09 & $1,944,641$ & $11,427,328$ & $329,366,378$ \\
\hline 10/30/09 & $1,944,641$ & $-6,508,934$ & $320,912,803$ \\
\hline $11 / 30 / 09$ & $1,944,641$ & $18,408,841$ & $337,377,002$ \\
\hline $12 / 31 / 09$ & $1,944,641$ & $5,995,391$ & $341,427,752$ \\
\hline
\end{tabular}




\begin{tabular}{|c|c|c|c|}
\hline $01 / 29 / 10$ & $1,944,641$ & $-12,624,039$ & $326,859,072$ \\
\hline $02 / 26 / 10$ & $1,944,641$ & $9,319,959$ & $334,234,390$ \\
\hline $03 / 31 / 10$ & $1,944,641$ & $19,651,768$ & $351,941,516$ \\
\hline $04 / 30 / 10$ & $1,944,641$ & $5,194,420$ & $355,191,295$ \\
\hline $05 / 28 / 10$ & $1,944,641$ & $-29,117,132$ & $324,129,522$ \\
\hline $06 / 30 / 10$ & $1,944,641$ & $-17,464,869$ & $304,720,012$ \\
\hline $07 / 30 / 10$ & $2,031,467$ & $20,957,982$ & $323,646,527$ \\
\hline $08 / 31 / 10$ & $2,031,467$ & $-15,356,757$ & $306,258,303$ \\
\hline 09/30/10 & $2,031,467$ & $26,813,253$ & $331,040,089$ \\
\hline $10 / 29 / 10$ & $2,031,467$ & $12,200,794$ & $341,209,416$ \\
\hline $11 / 30 / 10$ & $2,031,467$ & $-781,466$ & $338,396,483$ \\
\hline $12 / 31 / 10$ & $2,031,467$ & $22,097,315$ & $358,462,331$ \\
\hline $01 / 31 / 11$ & $2,031,467$ & $8,117,591$ & $364,548,455$ \\
\hline $02 / 28 / 11$ & $2,031,467$ & $11,649,723$ & $374,166,711$ \\
\hline $03 / 31 / 11$ & $2,031,467$ & $-391,866$ & $371,743,379$ \\
\hline $04 / 29 / 11$ & $2,031,467$ & $10,592,961$ & $380,304,873$ \\
\hline $05 / 31 / 11$ & $2,031,467$ & $-5,134,469$ & $373,138,938$ \\
\hline $06 / 30 / 11$ & $2,031,467$ & $-6,812,587$ & $364,294,884$ \\
\hline 07/29/11 & $2,428,633$ & $-7,823,027$ & $354,043,224$ \\
\hline 08/31/11 & $2,428,633$ & $-20,106,503$ & $331,508,088$ \\
\hline 09/30/11 & $2,428,633$ & $-23,789,688$ & $305,289,768$ \\
\hline $10 / 31 / 11$ & $2,428,633$ & $32,886,741$ & $335,747,876$ \\
\hline $11 / 30 / 11$ & $2,428,633$ & $-1,698,429$ & $331,620,814$ \\
\hline $12 / 30 / 11$ & $2,428,633$ & $2,829,638$ & $332,021,820$ \\
\hline $01 / 31 / 12$ & $2,428,633$ & $14,470,512$ & $344,063,699$ \\
\hline 02/29/12 & $2,428,633$ & $13,965,356$ & $355,600,423$ \\
\hline $03 / 30 / 12$ & $2,428,633$ & $11,141,806$ & $364,313,597$ \\
\hline $04 / 30 / 12$ & $2,428,633$ & $-2,731,440$ & $359,153,524$ \\
\hline $05 / 31 / 12$ & $2,428,633$ & $-22,501,209$ & $334,223,682$ \\
\hline 06/29/12 & $2,428,633$ & $13,220,191$ & $345,015,241$ \\
\hline $07 / 31 / 12$ & $2,300,102$ & $4,346,377$ & $347,061,517$ \\
\hline 08/31/12 & $2,300,102$ & $6,859,102$ & $351,620,518$ \\
\hline 09/28/12 & $2,300,102$ & $8,521,907$ & $357,842,323$ \\
\hline $10 / 31 / 12$ & $2,300,102$ & $-7,081,486$ & $348,460,735$ \\
\hline $11 / 30 / 12$ & $2,300,102$ & 991,964 & $347,152,597$ \\
\hline $12 / 31 / 12$ & $2,300,102$ & $2,453,782$ & $347,306,278$ \\
\hline $01 / 31 / 13$ & $2,300,102$ & $17,513,983$ & $362,520,160$ \\
\hline $02 / 28 / 13$ & $2,300,102$ & $4,009,692$ & $364,229,750$ \\
\hline $03 / 28 / 13$ & $2,300,102$ & $13,107,827$ & $375,037,475$ \\
\hline $04 / 30 / 13$ & $2,300,102$ & $6,782,839$ & $379,520,213$ \\
\hline $05 / 31 / 13$ & $2,300,102$ & $7,879,896$ & $385,100,007$ \\
\hline $06 / 28 / 13$ & $2,300,102$ & $-5,776,240$ & $377,023,665$ \\
\hline $07 / 31 / 13$ & $2,513,491$ & $18,648,386$ & $393,158,561$ \\
\hline $08 / 30 / 13$ & $2,513,491$ & $-12,305,082$ & $378,339,988$ \\
\hline $09 / 30 / 13$ & $2,513,491$ & $11,255,416$ & $387,081,913$ \\
\hline $10 / 31 / 13$ & $2,513,491$ & $17,262,212$ & $401,830,634$ \\
\hline $11 / 29 / 13$ & $2,513,491$ & $11,271,133$ & $410,588,275$ \\
\hline $12 / 31 / 13$ & $2,513,491$ & $9,674,623$ & $417,749,407$ \\
\hline $01 / 31 / 14$ & $2,513,491$ & $-14,864,733$ & $400,371,183$ \\
\hline $02 / 28 / 14$ & $2,513,491$ & $17,262,819$ & $415,120,511$ \\
\hline $03 / 31 / 14$ & $2,513,491$ & $2,877,681$ & $415,484,700$ \\
\hline $04 / 30 / 14$ & $2,513,491$ & $2,576,336$ & $415,547,546$ \\
\hline $05 / 30 / 14$ & $2,513,491$ & $8,739,082$ & $421,773,137$ \\
\hline $06 / 30 / 14$ & $2,513,491$ & $8,038,285$ & $427,297,930$ \\
\hline $07 / 31 / 14$ & $2,848,653$ & $-6,443,594$ & $418,005,683$ \\
\hline
\end{tabular}




$\begin{array}{lrrr}08 / 29 / 14 & 2,848,653 & 15,740,138 & 430,897,169 \\ 09 / 30 / 14 & 2,848,653 & -6,684,878 & 421,363,638 \\ 10 / 31 / 14 & 2,848,653 & 9,776,250 & 428,291,235 \\ 11 / 28 / 14 & 2,848,653 & 10,507,519 & 435,950,101 \\ 12 / 31 / 14 & 2,848,653 & -1,825,982 & 431,275,466 \\ 01 / 30 / 15 & 2,848,653 & -13,387,156 & 415,039,657 \\ 02 / 27 / 15 & 2,848,653 & 22,782,567 & 434,973,571 \\ 03 / 31 / 15 & 2,848,653 & -7,566,825 & 424,558,094 \\ 04 / 30 / 15 & 2,848,653 & 3,617,559 & 425,327,000\end{array}$

Figure $8.8 \%$ Spending, based on prior year's endowment balance

When the actual S\&P 500 monthly returns are applied to the initial $\$ 500,000,000$ endowment balance, using each of the spending rates applied to the prior month and prior year balances, respectively, the ending endowment balances are as follows:

\begin{tabular}{|c|c|c|}
\hline \multicolumn{3}{|c|}{ Applied to } \\
\hline Spending Rate & Prior Month & $\underline{\text { Prior Year }}$ \\
\hline $0 \%$ & $\$ 875,161,561$ & $\$ 875,161,561$ \\
\hline $2 \%$ & $\$ 718,132,588$ & $\$ 734,754,918$ \\
\hline $5 \%$ & $\$ 533,470,624$ & $\$ 561,427,941$ \\
\hline $8 \%$ & $\$ 395,997,524$ & $\$ 425,327,000$ \\
\hline
\end{tabular}

We then attempted to develop a single function to estimate the ending endowment balance, making use of specific metrics generally considered crucial to the determination of a portfolio balance:

(1) the mean return

(2) the standard deviation of the returns (as a measure of volatility)

(3) the spending rate

We realized that it might be of some value to incorporate the spending pattern into the formula as well (that is, whether spending was based on the prior month's or rather the prior year's balance), but in this preliminary research wished to see how well the function performed without specifying the spending pattern.

A number of studies -- for example, Jacquier, Kane, and Marcios (2003), Blume (1974), and Indro and Lee (1997) have discussed the issue of whether one should use the arithmetic mean or instead the geometric mean (which takes compounding into account) when examining expected long-run rates of return and/or forecasting the terminal value of a portfolio. Blitz and van Vliet (2007) conducted a study using twenty years' worth of data to demonstrate the effect of volatility on a portfolio balance, and Marx and Hargis (2014) discussed the effect of volatility as it affects investors taking withdrawals from a portfolio during retirement. Many researchers (among them Finke, Pfau, and Blanchett (2013), Vernon (2014), and Guyton (2004)) have concentrated specifically on the effect of spending from a portfolio, usually in the context of systematic withdrawals for retirement income.

There was some trial and error involved in the development of the estimating function ultimately used, especially as it related to the utilization of a constant, attached as a weight to the average return. The formula ultimately used to make our predictions of the final balances is as follows:

$$
\mathrm{EB}=(\mathrm{BB} *(1+(\mathrm{SD} / \mathrm{C} * \mathrm{MR}) * .01)-\mathrm{SR}) \wedge(\mathrm{N} / 12)
$$

where

$\mathrm{EB}=$ ending portfolio balance

$\mathrm{BB}=$ beginning portfolio balance

$\mathrm{SD}=$ standard deviation of the monthly returns

$\mathrm{MR}=$ arithmetic mean of the monthly returns

$\mathrm{SR}=$ spending rate 
$\mathrm{N}=$ number of periods

$\mathrm{C}=$ constant

Note that the formula above indeed does incorporate the mean return, the standard deviation of the returns, and the spending rate. In fact, it makes use of a modified version of the coefficient of variation (standard deviation/mean), used to measure relative volatility. Here a weight or adjustment factor was applied to the mean. The weight used (C $=.4125$ ) was the one that provided the "best fit" (based on trial and error) in terms of how close the predicted final portfolio balances using the formula were to what the actual portfolio balances would have been. Note that "best fit" is in quotations because we did not determine the optimal constant value based on a specific statistical test for goodness of fit.

The calculated ending balances using our function compared to the actual ending balances when spending is based on the prior month's portfolio balance are as follows:

\begin{tabular}{|c|c|c|}
\hline Spending Rate & $\underline{\text { Actual }}$ & Calculated \\
\hline $0 \%$ & $\$ 875,161,561$ & $\$ 877,361,623$ \\
\hline $2 \%$ & $\$ 718,132,588$ & $\$ 726,127,708$ \\
\hline $5 \%$ & $\$ 533,470,624$ & $\$ 542,935,738$ \\
\hline $8 \%$ & $\$ 395,997,524$ & $\$ 402,411,1$ \\
\hline
\end{tabular}

The calculated ending balances using the function compared to the actual ending balances when spending is based on the prior year's portfolio balance are as follows:

\begin{tabular}{|c|c|c|}
\hline Spending Rate & Actual & $\underline{\text { Calculated }}$ \\
\hline $0 \%$ & $\$ 875,161,561$ & $\$ 877,361,623$ \\
\hline $2 \%$ & $\$ 734,754,918$ & $\$ 726,127,708$ \\
\hline $5 \%$ & $\$ 561,427,941$ & $\$ 542,935,738$ \\
\hline $8 \%$ & $\$ 425,327,000$ & $\$ 402,411,100$ \\
\hline
\end{tabular}

\section{Analysis}

The impetus for this research was the suggestion that an institution that bases its spending on a prior month's or prior year's balance will be harmed when markets are volatile, because it may be spending based on a higher previous portfolio balance at a time when a market down cycle is further eroding the corpus. That would make it more difficult for the portfolio to recover to prior levels (the math of investing is basic to all investors - if the portfolio drops $50 \%$, then it needs to increase by $100 \%$ to get back to where it started. If the portfolio increases by $50 \%$, then it only needs to drop by $33 \%$ get back to the starting point). An examination of the formula used in this study (which seems to do a decent job of estimating final portfolio balances) would lead one to a different conclusion regarding volatility, as evidenced by the predicted ending balances being rather good estimates of the actual, without consideration of the smoothing of spending.

Relationships clearly change over time. Therefore, the adjustment factor (applied to the average return) providing the best fit for a different time period would be expected to vary from the one for the ten year period under consideration here. However, if the general form of the relationship is shown to be robust over time, investors may well be able to be less concerned about volatility (as measured by the standard deviation) than they currently are.

Given that the formula does not include a variable that takes into account the spending pattern, but rather only one for the spending rate, the formula predicts the same ending balance for both patterns used (spending based on the prior month's balance and spending based on the prior year's balance). Therefore, if the formula is indeed a decent predictor in both cases, then perhaps the spending pattern makes little difference in the ending portfolio balance. However, clearly more research would be required using broader differences in the spending pattern to see the extent to which that result holds.

\section{Additional Test}

In order to test whether the formula is indeed robust, we took the data set and arbitrarily truncated the data. The original data set had 119 observations. This was truncated to 98 observations for spending based on the prior year's balance and 88 observations for spending based on the prior month's balance. Then the formula described earlier in this paper was applied. The arithmetic mean return and standard deviation of the returns were $0.5111 \%$ and $4.6314 \%$, 
respectively, for spending based on the prior year's balance and $0.4947 \%$ and $4.8075 \%$ for spending based on the prior month's balance.

The calculated ending balances employing our function (still using the original adjustment factor of .4125) compared to the actual ending balances when spending is based on the prior month's portfolio balance are as follows:

\begin{tabular}{|c|c|c|}
\hline Spending Rate & Actual & $\underline{\text { Calculated }}$ \\
\hline $0 \%$ & $\$ 783,076,676$ & $\$ 754,211,200$ \\
\hline $2 \%$ & $\$ 667,009,974$ & $\$ 655,680,083$ \\
\hline $5 \%$ & $\$ 535,526,649$ & $\$ 528,758,210$ \\
\hline $8 \%$ & $\$ 429,725,728$ & $\$ 423,641,059$ \\
\hline
\end{tabular}

The calculated ending balances using the function compared to the actual ending balances when spending is based on the prior year's portfolio balance are as follows:

\begin{tabular}{|c|c|c|}
\hline Spending Rate & Actual & Calculated \\
\hline $0 \%$ & $\$ 824,032,894$ & $\$ 788,627,972$ \\
\hline $2 \%$ & $\$ 702,447,450$ & $\$ 674,752,489$ \\
\hline $5 \%$ & $\$ 549,609,947$ & $\$ 530,963,277$ \\
\hline $8 \%$ & $\$ 426,825,583$ & $\$ 414,798,943$ \\
\hline
\end{tabular}

It should be noted that a modest change of the adjustment factor from .4125 to .38 for the truncated data results in the ending balance being somewhere between $98 \%$ and $101 \%$ of the predicted balance for all eight scenarios.

\section{Conclusions}

Fiduciaries who are entrusted with the management of an endowment have an obligation, moral and legal, to manage that endowment to the goals specified in the Investment Policy Statement. Often, the first goal is "perpetuity." Further goals may include absolute return targets (say 6\%), and/or relative return targets (S\&P 500 plus 5\% or top quartile among endowments of $\$ 1$ billion and over). A critical estimate is the amount of the organization's spending budget. Frequently a rolling twelve quarters is used, with a set percentage taken for spending. This percentage can be regulatory (5\% in the case of private foundations), contractual (built into the pension model) or organizationally specific (a University's spending policy). Using a set percentage based on the past might lead to spending that cannot be sustained, jeopardizing perpetuity. In a sustained market drop, spending will be based on the previous three years' average portfolio balance (where the rolling twelve quarters is used), resulting in more spending than the current portfolio balance can fund.

This paper develops a robust function that predicts the ending portfolio balance given a set of variable inputs. A fiduciary can adjust the variables to accommodate future scenarios, allowing them to see the resultant effect on the ending balance of the portfolio. This would allow for adjustments to controllable variables, such as spending, in advance of the permanent decline of the corpus.

As a method of obtaining a rough estimate of a portfolio's ending balance, the function developed in this study seems to work well. When applied to sixteen scenarios, the actual ending balances as a proportion of the predicted ending balances were (from lowest to highest): 


\subsection{4 \\ 1.04 \\ 1.04 \\ 1.06}

The results above were obtained from our function by applying the same adjustment factor to the average return for all sixteen scenarios (which made use of returns for as few as 88 months to as many as 119 months). Therefore, we are in a sense providing a conservative estimate of the function's efficiency, given that one might expect to alter the adjustment factor (to obtain the best fit) when changing the time period under consideration. We see also that the function works well in each spending scenario, regardless as to whether the spending is calculated based on the prior month's or prior year's balance.

Any person or organization that has an investment fund, portfolio, or endowment where they have access to historical return mean and standard deviation, and have used a standard rate of spending can apply the function to see how well it estimates the ending balance. If it turns out to be a good predictor, the function can be used to estimate future balances based on the arithmetic mean return and the standard deviation of the returns. This could be useful, for instance, to predict how the portfolio will withstand an increase in the spending rate. Furthermore, as suggested earlier, confidence in the function presented here could lead to a decrease in one's trepidation about volatility of returns.

Future research should incorporate the spending pattern as part of the formula. This would perhaps yield forecasted balances that were even closer to the observed actual balances. It might also be of value to develop a series of return mean-standard deviation pairings to understand how a change in one might result in the change in the other. A casual look at our formula might lead one to conclude that the ending portfolio balance can be increased merely by increasing the standard deviation. This would be an ill-advised implication, given that the required condition "holding all else constant" is not possible - return mean and the standard deviation of returns are linked.

\section{References}

Blitz, D., \& van Vliet, P. (2007). The volatility effect. Journal of Portfolio Management, 34, 102-113. http://dx.doi.org/10.3905/jpm.2007.698039

Blume, M. E. (1974). Unbiased estimation of long-run expected rates of return. Journal of American Statistical Association, 69(347), 634-638. http://dx.doi.org/10.1080/01621459.1974.10480180

Finke, M. S., Pfau, W. D., \& Blanchett, D. M. (2013). The 4 percent rule is not safe in a low-yield world. Journal of Financial Planning, 26(6), 46-55. http://dx.doi.org/10.2139/ssrn.2201323

Guyton, J. T. (2004). Decision rules and portfolio management for retirees: is the safe initial withdrawal rate too safe? Journal of Financial Planning, 17(10), 54-62.

Haber, J. (2014a). Food for thought: which would you prefer, frontloaded positive returns, or staggered? Retrieved from www.investorintelligencenetwork.com

Haber, J. (2014b). Endowment controller offers up mathematical food for thought. Retrieved from www.investorintelligencenetwork.com

Indro, D. C., \& Lee, W. Y. (1997). Biases in arithmetic and geometric averages as estimates of long run expected rates of return and risk premia. Financial Management, 26(4), 81-90. http://dx.doi.org/10.2307/3666130

Jacquier, E., Kane, A., \& Marcios, A. J. (2003). Geometric or arithmetic mean: a reconsideration. Financial Analysts Journal, 59(6), 46-53. http://dx.doi.org/10.2469/faj.v59.n6.2574

Marx, C., \& Hargis, K. (2014). Volatility in retirement - what a drag! CONTEXT - the AB (Alliance Bernstein) Blog on Investing, August 7. 